\title{
IMBALANCED WEAK MAGNETOHYDRODYNAMIC TURBULENCE
}

\author{
Yoram Lithwick AND Peter GoldReich \\ 130-33 California Institute of Technology, Pasadena, CA 91125; yoram@tapir.caltech.edu,pmg@gps.caltech.edu \\ Received 2002 July 27; accepted 2002 September 10
}

\begin{abstract}
Weak MHD turbulence consists of waves that propagate along magnetic field lines, in both directions. When two oppositely directed waves collide, they distort each other, without changing their respective energies. Each wave suffers many collisions before cascading; by contrast, in strong MHD turbulence, waves cascade on the same timescale at which they collide. "Imbalance" means that more energy is going in one direction than the other. In general, MHD turbulence is imbalanced. Yet imbalanced MHD cascades are not understood. For example, turbulence in the solar wind is observed to be imbalanced, so solar wind turbulence will not be understood until a theory of the imbalanced cascade is developed. We solve weak MHD turbulence that is imbalanced. Of crucial importance is that the energies going in both directions are forced to equalize at the dissipation scale. This "pinning" of the energy spectra was discovered by Grappin and coworkers. It affects the entire inertial range. Weak MHD turbulence is particularly interesting because perturbation theory is applicable. Hence, it can be described with a simple kinetic equation. Galtier and coworkers derived this kinetic equation. We present a simpler, more physical derivation, based on the picture of colliding wavepackets. In the process, we clarify the role of the zero-frequency mode. We also explain why Goldreich \& Sridhar claimed that perturbation theory is inapplicable, and why this claim is wrong. (Our "weak" is equivalent to Goldreich \& Sridhar's "intermediate.") We perform numerical simulations of the kinetic equation to verify our claims. We construct simplified model equations that illustrate the main effects. Finally, we show that a large magnetic Prandtl number does not have a significant effect, and that hyperviscosity leads to a pronounced bottleneck effect.
\end{abstract}

Subject headings: MHD — turbulence

\section{INTRODUCTION}

MHD turbulence is ubiquitous in astrophysics. For example, it is present in the Sun, the solar wind, the interstellar medium, molecular clouds, accretion disks, and galaxy clusters. Theoretical understanding of incompressible MHD turbulence has grown explosively in the last decade. Nonetheless, it remains underdeveloped.

Iroshnikov (1963) and Kraichnan (1965) developed a theory for MHD turbulence. They realized that the magnetic field at the largest length scale in a cascade directly affects all of the smaller length scales. Small-scale fluctuations can be treated as small-amplitude waves in the presence of a large mean magnetic field. By contrast, the large-scale velocity is unimportant for small-scale dynamics; it can be eliminated by a change of variables, since the equations of MHD are invariant under Galilean transformations. ${ }^{1}$

Despite their realization of the importance of the mean magnetic field, Iroshnikov and Kraichnan assumed that smallscale fluctuations are isotropic. Numerical simulations later showed that this assumption is wrong. Even with isotropic excitation at large scales, fluctuations on smaller scales are elongated along the mean magnetic field (e.g., Montgomery \& Turner 1981; Shebalin, Matthaeus, \& Montgomery 1983).

In retrospect, it is not very surprising to find elongated fluctuations. Arbitrary disturbances in incompressible MHD can be decomposed into shear-Alfvén and pseudoAlfvén waves. Each wave travels either up or down the mean field at the Alfvén speed, $v_{\mathrm{A}}$, which is the magnitude of the mean field in velocity units. Consider stirring a magnetofluid with a spoon that is moving at speed $v_{\lambda} \ll v_{\text {A }}$, for a time comparable to the spoon's width $\lambda$ divided by $v_{\lambda}{ }^{2}$

\footnotetext{
${ }^{1}$ One outgrowth of Iroshnikov and Kraichnan's theory that is particularly relevant to the present paper is the investigation of imbalanced MHD turbulence by Grappin et al. (1983). We discuss it in $\S 3.3$.

${ }^{2}$ In a turbulent cascade, one would expect $v_{\lambda} \ll v_{\mathrm{A}}$ on small scales, since $v_{\lambda}$ decreases toward small scales, whereas $v_{\mathrm{A}}$ is unchanged.
}

Alfvén waves are radiated away from the spoon, parallel to the mean field with speed $\pm v_{\mathrm{A}}$. After the disturbance is finished, there are two wavepackets travelling away from each other. Each wavepacket is elongated along the mean field, with parallel-to-transverse aspect ratio $\sim v_{\mathrm{A}} / v_{\lambda} \gg 1$.

The characteristics of MHD turbulence depend critically on the amount of elongation. When parallel-to-transverse aspect ratios are smaller than $v_{\mathrm{A}} / v_{\lambda}$, waves collide many times before cascading. Hence the turbulence is "weak," and perturbation theory can be used to derive a kinetic equation and a spectrum (Sridhar \& Goldreich 1994; see Zakharov, L'vov, \& Falkovich 1992 for a general review of weak turbulence). Goldreich \& Sridhar (1997) and Ng \& Bhattacharjee (1997) deduced the spectrum of the balanced weak cascade from scaling arguments. ${ }^{3}$ However, similar

\footnotetext{
${ }^{3}$ We relegate some of the history to a footnote because it can be confusing. Sridhar \& Goldreich (1994) developed the first theory of MHD turbulence that accounted for the anisotropy of fluctuations. They claimed that "three-wave" processes vanish in weak MHD turbulence, and "fourwave" processes must be considered (i.e., perturbation theory is trivial to first order, so second-order terms are important). As a result, they used four-wave couplings to derive a kinetic equation and a spectrum for weak MHD turbulence. Montgomery \& Matthaeus (1995) claimed, and Ng \& Bhattacharjee (1996) showed, that Sridhar \& Goldreich (1994) are wrong, and three-wave processes do not vanish. Goldreich \& Sridhar (1997) explained the contradiction: Sridhar \& Goldreich (1994) had unknowingly assumed that field-line wander is limited, i.e., that the separation between any two field lines is nearly constant along their entire length; in this case, three-wave couplings are negligible and the kinetic equation based on fourwave couplings is correct. In the more realistic case in which field lines do wander, three-wave processes are important. Goldreich \& Sridhar (1997) went on to argue that, in the latter case, perturbation theory is inappropriate, and couplings of all order are of comparable magnitude; so they called this "intermediate" turbulence. Galtier et al. (2000) argued that perturbation theory is appropriate, even when three-wave processes are important. In the Appendix of the present paper, we use Goldreich \& Sridhar's picture of wavepackets following wandering field lines to clarify the controversy, and to explain why perturbation theory works. Because it does work, we call the cascade "weak" instead of "intermediate."
} 
scaling arguments are inadequate for the imbalanced cascade (see $\S 3.2$ of the present paper). Galtier et al. (2000) derived the kinetic equation for the weak imbalanced cascade. Their balanced spectrum agrees with that of Goldreich \& Sridhar (1997) and Ng \& Bhattacharjee (1997). They also presented a partial solution for the general imbalanced case. In $\S 3.2$, we explain why their solution is incomplete; in $\S 4$ we give the complete solution.

Even if aspect ratios are smaller than $v_{\mathrm{A}} / v_{\lambda}$ on large scales, at a small enough scale they become comparable to $v_{\mathrm{A}} / v_{\lambda}$. Below this scale perturbation theory breaks down, and weak turbulence becomes "strong." Goldreich \& Sridhar (1995) worked out the scalings for the balanced strong cascade. They argued that aspect ratios are comparable to $v_{\mathrm{A}} / v_{\lambda}$ at all scales in the strong regime. Strong turbulence is difficult, largely because it is nonperturbative. Although strong and weak turbulence differ in a number of ways, they also share many similarities. One of our motivations for studying weak turbulence is to gain insight into strong turbulence. In particular, turbulence in the solar wind is observed to be imbalanced; it cannot be understood without a theory for imbalanced strong MHD turbulence. Yet this theory is unknown. In a future paper, we will work it out by extending the results of the present paper.

\section{BASIC EQUATIONS}

Ideal incompressible $\mathrm{MHD}^{4}$ is described by the following equations of motion:

$$
\begin{gathered}
\partial_{t} \boldsymbol{v}+\boldsymbol{v} \cdot \boldsymbol{\nabla} \boldsymbol{v}=-\boldsymbol{\nabla} P+\boldsymbol{B} \cdot \boldsymbol{\nabla} \boldsymbol{B}, \\
\partial_{t} \boldsymbol{B}+\boldsymbol{v} \cdot \boldsymbol{\nabla} \boldsymbol{B}=\boldsymbol{B} \cdot \boldsymbol{\nabla} \boldsymbol{v}, \\
\boldsymbol{\nabla} \cdot \boldsymbol{v}=\boldsymbol{\nabla} \cdot \boldsymbol{B}=0 .
\end{gathered}
$$

The density is set to unity; the fluid velocity is $\boldsymbol{v}$; the magnetic field in velocity units is $\boldsymbol{B} \equiv$ (magnetic field) $/(4 \pi)^{1 / 2}$; and the total pressure is $P \equiv p+B^{2} / 2$, which is the sum of the thermal and magnetic pressures. Viscous and resistive terms are neglected in the above equations; they are important on small scales, and will be included where required.

We decompose the magnetic field into its mean, $v_{\mathrm{A}} \hat{\boldsymbol{z}}$, where $v_{\mathrm{A}}$ is the Alfvén speed and $\hat{\boldsymbol{z}}$ is a unit vector, and into its fluctuating part $\boldsymbol{b} \equiv \boldsymbol{B}-v_{\mathrm{A}} \hat{\boldsymbol{z}}$. With this decomposition, the equations of motion can be written in terms of the Elsasser variables, $\boldsymbol{w}^{\uparrow} \equiv \boldsymbol{v}-\boldsymbol{b}$ and $\boldsymbol{w} \downarrow \equiv \boldsymbol{v}+\boldsymbol{b}$, as

$$
\begin{gathered}
\partial_{t} \boldsymbol{w}^{\uparrow}+v_{\mathrm{A}} \partial_{z} \boldsymbol{w}^{\uparrow}=-\boldsymbol{w}^{\downarrow} \cdot \boldsymbol{\nabla} \boldsymbol{w}^{\uparrow}-\nabla P, \\
\partial_{t} \boldsymbol{w}^{\downarrow}-v_{\mathrm{A}} \partial_{z} \boldsymbol{w}^{\downarrow}=-\boldsymbol{w}^{\uparrow} \cdot \boldsymbol{\nabla} \boldsymbol{w}^{\downarrow}-\nabla P, \\
\boldsymbol{\nabla} \cdot \boldsymbol{w}^{\uparrow}=\boldsymbol{\nabla} \cdot \boldsymbol{w}^{\downarrow}=0 .
\end{gathered}
$$

Note that $P$ is not an independent degree of freedom. Taking the divergence of either equation (4) or (5) yields

$$
P=-\nabla^{-2}\left(\boldsymbol{\nabla} \boldsymbol{w}^{\uparrow}: \nabla \boldsymbol{w}^{\downarrow}\right),
$$

where $\nabla^{-2}$ is the inverse Laplacian. When $\boldsymbol{w}^{\downarrow}=0, \boldsymbol{w}^{\uparrow}$ propagates undistorted upward along the mean magnetic field with speed $v_{\mathrm{A}}$. Similarly, when $\boldsymbol{w}^{\uparrow}=0, \boldsymbol{w} \downarrow$ propagates downward at $v_{\mathrm{A}}$. Nonlinear interactions occur only between

\footnotetext{
${ }^{4}$ In this paper, we consider only incompressible MHD turbulence; compressibility does not alter the dynamics very much (Lithwick \& Goldreich 2001).
}

oppositely directed wavepackets. It is these interactions that are responsible for turbulence.

There are three conserved quantities in incompressible MHD. Two of these are immediately apparent from equations (4)-(6): the energies of the upgoing and of the downgoing waves, i.e., $\left(\boldsymbol{w}^{\uparrow}\right)^{2}$ and $(\boldsymbol{w} \downarrow)^{2}$. Technically, these are twice the energy per unit mass. We refer to them as simply energies throughout the paper. These energies are directly related to the total (kinetic plus magnetic) energy $\propto\left(\boldsymbol{w}^{\uparrow}\right)^{2}+\left(\boldsymbol{w}^{\downarrow}\right)^{2}$ and to the cross-helicity $\propto\left(\boldsymbol{w}^{\uparrow}\right)^{2}$ $-\left(w^{\downarrow}\right)^{2}$. The focus of this paper is turbulence where the energies in the up and down waves differ, or, equivalently, where the cross-helicity is nonzero. The third conserved quantity is magnetic helicity; however, we consider only nonhelical turbulence in this paper, so helicity does not play a role.

In MHD turbulence, on length scales much smaller than the outer scale, there is effectively a strong mean magnetic field that is due to fluctuations on the largest length scales. Gradients transverse to this mean field are much larger than gradients along it (e.g., Shebalin et al. 1983; Goldreich \& Sridhar 1995, 1997; Ng \& Bhattacharjee 1996). This allows the MHD equations to be slightly simplified. Denoting transverse components with the symbol $\perp \equiv(x, y)$, the transverse components of equation (4) are

$$
\partial_{t} \boldsymbol{w}_{\perp}^{\uparrow}+v_{\mathrm{A}} \partial_{z} \boldsymbol{w}_{\perp}^{\uparrow} \approx-\boldsymbol{w}_{\perp}^{\downarrow} \cdot \nabla_{\perp} \boldsymbol{w}_{\perp}^{\uparrow}-\nabla_{\perp} P,
$$

assuming that $w_{z}^{\downarrow} \partial_{z} \boldsymbol{w}^{\uparrow}$ is much smaller than $\boldsymbol{w}_{\perp}^{\downarrow} \cdot \nabla_{\perp} \boldsymbol{w}^{\uparrow}$, and equation (6) is

$$
\nabla_{\perp} \cdot \boldsymbol{w}_{\perp}^{\uparrow} \approx 0
$$

We assume that the parallel components of $\boldsymbol{w}^{\uparrow}$ and $\boldsymbol{w} \downarrow$ are either comparable to or less than their respective perpendicular components. We will see below that this is typically the case in the inertial range of a turbulent cascade. Similarly,

$$
\begin{gathered}
\partial_{t} \boldsymbol{w}_{\perp}^{\downarrow}-v_{\mathrm{A}} \partial_{z} \boldsymbol{w}_{\perp}^{\downarrow} \approx-\boldsymbol{w}_{\perp}^{\uparrow} \cdot \nabla_{\perp} \boldsymbol{w}_{\perp}^{\downarrow}-\nabla_{\perp} P, \\
\boldsymbol{\nabla}_{\perp} \cdot \boldsymbol{w}_{\perp}^{\downarrow} \approx 0 .
\end{gathered}
$$

If we change $\approx$ to $=$, equations (8)-(11) form a closed set. They are called the equations of reduced MHD. They apply also in compressible MHD whenever transverse gradients are larger than parallel ones (e.g., Biskamp 1995). The main goal of this paper is to solve these equations. Although the complete equations are not much more complicated, it simplifies our discussions to neglect parallel gradients relative to perpendicular ones at the outset. There are two conserved energies in reduced MHD: $\left(\boldsymbol{w}_{\perp}^{\uparrow}\right)^{2}$ and $\left(\boldsymbol{w}_{\perp}^{\downarrow}\right)^{2}$.

The parallel components of equations (4) and (5) are

$$
\begin{aligned}
& \partial_{t} w_{z}^{\uparrow}+v_{\mathrm{A}} \partial_{z} w_{z}^{\uparrow} \approx-\boldsymbol{w}_{\perp}^{\downarrow} \cdot \nabla_{\perp} w_{z}^{\uparrow}, \\
& \partial_{t} w_{z}^{\downarrow}-v_{\mathrm{A}} \partial_{z} w_{z}^{\downarrow} \approx-\boldsymbol{w}_{\perp}^{\uparrow} \cdot \nabla_{\perp} w_{z}^{\downarrow},
\end{aligned}
$$

after neglecting parallel gradients relative to transverse ones, and after assuming that $\left|w_{z}^{\uparrow}\right|$ and $\left|w_{z}^{\downarrow}\right|$ are not much smaller than $\left|\boldsymbol{w}_{\perp}^{\uparrow}\right|$ and $\left|\boldsymbol{w}_{\perp}^{\downarrow}\right|$. Clearly, $\left(w_{z}^{\uparrow}\right)^{2}$ and $\left(w_{z}^{\downarrow}\right)^{2}$ are conserved quantities. The transverse equations describing reduced MHD are unaffected by these parallel equations because the former are independent of $w_{z}^{\uparrow}$ and $w_{z}^{\downarrow}$. Nonetheless, the parallel equations have observable consequences.

It is conventional to decompose the normal modes of linearized incompressible MHD, $\boldsymbol{w}^{\uparrow}$ and $\boldsymbol{w} \downarrow$, into shear-Alfvén and pseudo-Alfvén waves. These correspond to the Alfvén 
and slow waves of compressible MHD. When perpendicular gradients are much larger than parallel ones, $\boldsymbol{w}_{\perp}^{\uparrow}$ and $\boldsymbol{w}_{\perp}^{\downarrow}$ are nearly equivalent to shear-Alfvén waves; $w_{z}^{\uparrow}$ and $w_{z}^{\downarrow}$ are nearly equivalent to pseudo-Alfvén waves.

Also observationally relevant is the evolution of a passive scalar $s$, which satisfies $\partial_{t} s+\boldsymbol{v} \cdot \nabla s=0$. In terms of Elsasser variables, and after neglecting parallel gradients, the passive scalar satisfies

$$
\partial_{t} s \approx-\frac{1}{2}\left(\boldsymbol{w}_{\perp}^{\uparrow}+\boldsymbol{w}_{\perp}^{\downarrow}\right) \cdot \nabla_{\perp} s .
$$

To avoid a proliferation of subscripts, in the remainder of this paper we drop the $\perp$ from $\boldsymbol{w}_{\perp}^{\uparrow}$ and $\boldsymbol{w}_{\perp}^{\downarrow}$. To denote the parallel components, we use $w_{z}^{\uparrow}$ and $w_{z}^{\downarrow}$.

\section{WEAK MHD TURBULENCE: HEURISTIC DISCUSSION}

One of the virtues of weak MHD turbulence is that it can be analyzed in a mathematically rigorous way with perturbation theory; this yields a kinetic equation. Nevertheless, we begin with a qualitative description, which captures most of the features of the turbulent cascade.

\subsection{Scaling Relation}

MHD turbulence can be understood from the dynamics of $\boldsymbol{w}^{\uparrow}$ and $\boldsymbol{w}^{\downarrow}$ (eqs. [8]-[11] for reduced MHD, dropping $\perp$ subscripts). To linear order, $\boldsymbol{w}^{\uparrow}$ is a wave that propagates up the mean magnetic field lines at the Alfvén speed, $v_{\mathrm{A}} ; \boldsymbol{w}^{\downarrow}$ propagates down at $v_{\mathrm{A}}$. Each wave perturbs the mean magnetic field lines. Nonlinear terms describe the interaction between oppositely directed waves; each wave nearly follows the field lines perturbed by its collision partner. ${ }^{5}$

Consider an upgoing wavepacket that encounters a train of downgoing wavepackets. As the upgoing wave travels up the length of the downgoing train, it is gradually distorted. It tries to follow the perturbed field lines in the downgoing train, but these field lines "wander," i.e., the transverse separation between any two field lines changes. When the upwave has travelled through a sufficiently large number of downgoing wavepackets that the amount of field line wander is comparable to the up-wave's transverse size, then the up-wave cascades.

To be quantitative, let each downgoing wave in the train have a typical amplitude $w_{\lambda}^{\downarrow}$, a transverse size $\lambda$, and a parallel size $\Lambda$, where "transverse" and "parallel" refer to the orientation relative to the mean magnetic field. The most important collisions are between wavepackets of comparable transverse size (see $\S$ 4.3.1). So let the upgoing wave have transverse size $\lambda$ as well.

Since each downgoing wavepacket has a typical perturbed magnetic field of magnitude $\sim w_{\lambda}^{\downarrow}$ (neglecting the factor of $1 / 2)$, it bends the field lines by the angle $w_{\lambda}^{\downarrow} / v_{\mathrm{A}}$; the transverse displacement of a field line through this wavepacket is $\left(w_{\lambda}^{\downarrow} / v_{\mathrm{A}}\right) \Lambda$; and the wander of two typical field lines

5 The equation for a scalar quantity $f$ that travels upward at speed $v_{\mathrm{A}}$, while following the magnetic field lines of the downgoing $\boldsymbol{w}^{\downarrow}$, is $\left(\partial_{t}+v_{\mathrm{A}} \partial_{z}+\boldsymbol{w}^{\downarrow} \cdot \nabla_{\perp}\right) f=0$. Eq. (8) for the vector $\boldsymbol{w}^{\uparrow}$ differs from this because of the pressure term, which is required to keep $\boldsymbol{w}^{\uparrow}$ incompressible, while conserving the energy $\left(\boldsymbol{w}^{\dagger}\right)^{2}$. Thus, $\boldsymbol{w}^{\uparrow}$ does not exactly follow the field lines of $\boldsymbol{w} \downarrow$. Nonetheless, this deviation does not greatly affect the behavior of the turbulence. Dissipation is a second effect that prevents the following of field lines. In the present discussion, we consider length scales that are sufficiently large that dissipation can be neglected. through the wavepacket, if they are initially separated by $\lambda$, is also $\left(w_{\lambda}^{\downarrow} / v_{\mathrm{A}}\right) \Lambda$.

In weak turbulence, the wander through a single wavepacket is smaller than the wavepacket's transverse size,

$$
\frac{w_{\lambda}^{\downarrow}}{v_{\mathrm{A}}} \Lambda \ll \lambda \quad \text { and } \quad \frac{w_{\lambda}^{\uparrow}}{v_{\mathrm{A}}} \Lambda \ll \lambda .
$$

When these inequalities are not satisfied, strong turbulence is applicable; see $\S$ 4.3.2. Thus, in weak turbulence an upgoing wavepacket must travel through many downgoing ones before cascading. After $N$ downgoing wavepackets, field lines have wandered a distance $\sim N^{1 / 2}\left(w_{\lambda}^{\downarrow} / v_{\mathrm{A}}\right) \Lambda$, assuming that wavepackets are statistically independent. The upgoing wavepacket is fully distorted - and hence cascaded - when the field lines it is following wander a distance $\lambda$, i.e., when $N \sim\left(\lambda v_{\mathrm{A}} / \Lambda w_{\lambda}^{\downarrow}\right)^{2}$. Since each downgoing wavepacket is crossed in the time $\Lambda / v_{\mathrm{A}}$, the cascade time of the upgoing wavepacket is

$$
t_{\text {cas }}^{\uparrow} \sim\left(\frac{\lambda v_{\mathrm{A}}}{\Lambda w_{\lambda}^{\downarrow}}\right)^{2} \frac{\Lambda}{v_{\mathrm{A}}} \sim\left(\frac{\lambda}{w_{\lambda}^{\downarrow}}\right)^{2} \frac{v_{\mathrm{A}}}{\Lambda} .
$$

In this time, the upgoing wavepacket travels a distance $v_{\mathrm{A}} t_{\text {cas }}^{\uparrow}$, which is much larger than its own length, $\Lambda .{ }^{6}$ The head and the tail of the upgoing wavepacket are both distorted by the same downgoing wavepackets, so both head and tail undergo nearly the same distortion as they cascade. ${ }^{7}$ Consequently, as the upgoing wavepacket cascades to smaller transverse length scales, it does not cascade to smaller parallel ones:

$$
\Lambda=\text { scale independent . }
$$

A proof of this follows from the three-wave resonance relations (Shebalin et al. 1983; see also $\S$ A.5 of the present paper).

We calculate the steady-state energy spectra by using Kolmogorov's picture of energy flowing from large to small length scales. The energy in up-waves flows from length scales larger than $\lambda$ to those smaller than $\lambda$ at the rate

$$
\epsilon^{\uparrow} \sim \frac{\left(w_{\lambda}^{\uparrow}\right)^{2}}{t_{\mathrm{cas}}^{\uparrow}} \sim\left(\frac{w_{\lambda}^{\uparrow} w_{\lambda}^{\downarrow}}{\lambda}\right)^{2} \frac{\Lambda}{v_{\mathrm{A}}} .
$$

We call this simply the "flux." We define $\epsilon^{\uparrow}$ more precisely below (eq. [51]). In steady state, the flux must be independent of $\lambda$, so

$$
w_{\lambda}^{\uparrow} w_{\lambda}^{\downarrow} \propto \lambda .
$$

\subsection{Insufficiency of Scaling Arguments for the Imbalanced Cascade}

A balanced cascade has $w_{\lambda}^{\uparrow}=w_{\lambda}^{\downarrow} \equiv w_{\lambda}$. Its solution in steady state is simple: $w_{\lambda} \propto \lambda^{1 / 2}$ and $\epsilon^{\uparrow}=\epsilon^{\downarrow} \sim w_{\lambda}^{4} \Lambda / \lambda^{2} v_{\mathrm{A}}$

\footnotetext{
${ }^{6}$ We assume throughout this paper that the upgoing waves' parallel length scale is the same as that of the downgoing waves, $\Lambda$; the extension to the case when they differ is trivial, as long as the inequalities (15) are both satisfied, with the appropriate $\Lambda$ 's.

${ }^{7}$ The head of the upgoing wavepacket slightly distorts each downgoing wavepacket; so the downgoing wavepacket seen by the tail is slightly distorted relative to that seen by the head. Nonetheless, this back-reaction is a higher order correction that can be ignored in weak turbulence; see $\S$ A.1.
} 
(Goldreich \& Sridhar 1997; Ng \& Bhattacharjee 1997). However, if the cascade is imbalanced, a number of complications arise.

By the symmetry between up- and downgoing waves, the downgoing flux is given by the analog of equation (18):

$$
\epsilon^{\downarrow} \sim\left(\frac{w_{\lambda}^{\uparrow} w_{\lambda}^{\downarrow}}{\lambda}\right)^{2} \frac{\Lambda}{v_{\mathrm{A}}} .
$$

Because $\epsilon^{\uparrow}$ and $\epsilon^{\downarrow}$ both depend on the same combination of $w_{\lambda}^{\uparrow}$ and $w_{\lambda}^{\downarrow}$-namely, their product - the steady-state solution is nontrivial. Had this degeneracy not occurred, e.g., had we found

$\epsilon^{\uparrow} \sim \frac{\left(w_{\lambda}^{\uparrow}\right)^{2+\gamma}\left(w_{\lambda}^{\downarrow}\right)^{2-\gamma}}{\lambda^{2}} \frac{\Lambda}{v_{\mathrm{A}}}$ and $\epsilon^{\downarrow} \sim \frac{\left(w_{\lambda}^{\uparrow}\right)^{2-\gamma}\left(w_{\lambda}^{\downarrow}\right)^{2+\gamma}}{\lambda^{2}} \frac{\Lambda}{v_{\mathrm{A}}}$,

where $\gamma \neq 0$, then the solution would have been simple: $w_{\lambda}^{\uparrow} \propto w_{\lambda}^{\downarrow} \propto \lambda^{1 / 2}$ and $\left(\epsilon^{\uparrow} / \epsilon^{\downarrow}\right) \sim\left(w_{\lambda}^{\uparrow} / w_{\lambda}^{\downarrow}\right)^{2 \gamma}$, which follow from the constancy of $\epsilon^{\uparrow}$ and $\epsilon \downarrow$ with $\lambda$.

But in weak MHD turbulence $\gamma=0$ (eqs. [18] and [20]); constancy of $\epsilon^{\downarrow}$ with $\lambda$ is forced by the constancy of $\epsilon^{\uparrow}$, and does not yield new information. One implication is that scaling arguments are insufficient to determine the flux ratio $\epsilon^{\uparrow} / \epsilon^{\downarrow}$. Physically, any flux ratio should be possible. But without the dimensionless coefficients of equations (18) and (20), $\epsilon^{\uparrow} / \epsilon^{\downarrow}$ cannot be determined. The coefficients cannot be obtained from scaling arguments; they depend on the spectral slopes of $w_{\lambda}^{\uparrow}$ and $w_{\lambda}^{\downarrow}$ (which are related through $w_{\lambda}^{\uparrow} w_{\lambda}^{\downarrow} \propto \lambda$; eq. [19]). Galtier et al. (2000) calculated the coefficients using kinetic equations. We explain how in $\S$ A.4. Therefore, these authors were able to relate the flux ratio to the spectral slopes.

The arguments presented thus far are still insufficiently constraining. Equations (18) and (20) constrain only the product $w_{\lambda}^{1} w_{\lambda}^{\downarrow}$. There are seemingly an infinite number of solutions with given values of $\epsilon^{\uparrow}$ and $\epsilon^{\downarrow}$, since $w_{\lambda}^{\uparrow}$ can be multiplied by any constant as long as $w_{\lambda}^{\downarrow}$ is divided by this same constant. Furthermore, we expect on physical grounds that if the values of $w^{\uparrow}$ and $w_{\lambda}^{\downarrow}$ at a given length scale are fixed (instead of the values of $\epsilon^{\uparrow}$ and $\epsilon^{\downarrow}$ ), the cascade should be completely constrained; however, in this case the constancy of equations (18) and (20) leaves the $\lambda$-dependence of $w_{\lambda}^{\uparrow} / w_{\lambda}^{\uparrow}$ completely undetermined - even given the coefficients derived by Galtier et al. (2000). Do $w_{\lambda}^{\uparrow}$ and $w_{\lambda}^{\downarrow}$ cross? Are they cut off by dissipation at the same scale? All of these problems for the imbalanced cascade can be resolved once the dynamics at the dissipation scale is understood.

\subsection{Dynamics at the Dissipation Scale: Pinned Spectra}

The main result of the present paper is that the energies of the up- and downgoing waves are forced to equalize - they are "pinned" - at the dissipation scale. This completely constrains the cascade. It is unusual that the dynamics at the dissipation scale has such an important influence. In this subsection we explain why pinning occurs. In $\S 4$, we give the resulting solution of the steady state cascade.

From equation (16), the cascade time of the upgoing waves is inversely proportional to the energy of the downgoing ones: $t_{\text {cas }}^{\uparrow}=\left(\lambda / w_{\lambda}^{\downarrow}\right)^{2}\left(v_{\mathrm{A}} / \Lambda\right)$, and similarly for the downgoing waves. We consider how the spectra evolve if initially, on length scales comparable to the dissipation scale, waves going in one direction are more energetic than the oppositely directed ones. To facilitate the discussion, we refer to Figure 1, which presents the results from a numerical simulation that we discuss in detail in $\S 6.2$. In the middle panels of Figures $1 a-1 d$, we plot $e^{\uparrow}(k) \sim\left(\lambda w_{\uparrow}^{\uparrow}\right)^{2}$ and $e^{\downarrow}(k) \sim\left(\lambda w_{\lambda}^{\downarrow}\right)^{2}$ as functions of wavenumber $k=1 / \lambda .^{8}$ The initial condition is shown in Figure 1a. Initially, $w_{\lambda}^{\uparrow}>w_{\lambda}^{\downarrow}$, so $t_{\text {cas }}^{\uparrow}>t_{\text {cas }}^{\downarrow}$. We consider a length-scale-dependent dissipation time, $t_{\text {diss }}$, that is the same for both up- and downgoing waves. ${ }^{9}$ On large length scales, the dissipation timescale is much longer than the cascade times; $t_{\text {diss }}$ decreases faster with increasing $k$ than both $t_{\text {cas }}^{\uparrow}$ and $t_{\text {cas }}^{t}$. The effects of dissipation are felt on length scales where $t_{\text {diss }}$ is comparable to - or smaller than-either $t_{\text {cas }}^{\uparrow}$ or $t_{\text {cas }}^{\downarrow}$. Since $t_{\text {cas }}^{\uparrow}>t_{\text {cas }}^{\downarrow}$ in the vicinity of the dissipation scale, the largest length scale at which dissipation effects are felt is where $t_{\text {cas }}^{\uparrow} \sim t_{\text {diss. }}$. In Figure $1 a$, this is at $k \sim 4000$. We now let the spectra evolve; see Figures $1 b-1 c$. We hold the energies at $k \simeq 1$ fixed; this does not affect the short-time behavior shown in Figures $1 b-1 c$. Since $w_{\lambda}^{\uparrow}$ feels the dissipative effects at $k \sim 4000$, its spectrum is exponentially cut off at smaller scales. This implies that the cascade time of the down-waves, $t_{\text {cas }}^{\downarrow}$, increases exponentially toward smaller scales. As a result, down-wave energy that is being cascaded from large to small scales cannot be cascaded fast enough at $k \gtrsim 4000$. Therefore, the down-waves' energy flux is backed up, and the $w_{\lambda}^{\downarrow}$ spectrum rises. Furthermore, as $w_{\lambda}^{\downarrow}$ rises, $t_{\text {cas }}^{\uparrow}$ falls, so the cascade time of the up-waves on small scales decreases, and the up-wave spectrum falls. The final result is that the two spectra are pinned at the dissipation scale. This pinning occurs very quickly, on the dissipation timescale.

Pinning was discovered by Grappin, Pouquet, \& Léorat (1983), when modelling imbalanced MHD turbulence with an eddy-damped quasi-normal Markovian (EDQNM) closure approximation. ${ }^{10}$ As with all closure approximations, their model equations are ad hoc, although physically motivated. By contrast, the weak turbulence kinetic equations of Galtier et al. (2000) that we use in the present paper can be derived directly from the MHD equations of motion using perturbation theory (as is done, for example, in the Appendix). Furthermore, Grappin et al.'s EDQNM approximation is based on the incorrect assumption that the turbulence is isotropic. As shown by Shebalin et al. (1983), this assumption is inconsistent with the resonance relations of weak MHD turbulence. Despite these caveats, the EDQNM equations of Grappin et al. share a number of similarities with the kinetic equations of weak MHD turbulence. Most importantly for the purposes of the present paper, Grappin et al. found from their model equations that scaling arguments constrained only the sum of the slopes of the up- and downgoing waves' spectra, and that the two spectra were constrained to be equal at the dissipation scale.

\section{STEADY-STATE ENERGY SPECTRA}

In steady state, the energy spectra $w_{\lambda}^{\uparrow}$ and $w_{\lambda}^{\downarrow}$ are power laws that (1) are pinned at the dissipation scale and (2)

\footnotetext{
${ }^{8}$ For the purposes of the present heuristic discussion, we consider $e^{\dagger}$ and $e^{\downarrow}$ to be equivalent to $\left(\lambda w_{\lambda}^{\uparrow}\right)^{2}$ and $\left(\lambda w_{\lambda}^{\downarrow}\right)^{2}$, respectively. The quantities $e^{\uparrow}$ and $e^{\downarrow}$ are defined more precisely below (eqs. [44] and [45]).

${ }^{9}$ For example, in the simulation presented in Fig. $1, t_{\text {diss }} \simeq \lambda^{2} / \nu$, where $\nu$ is both the viscosity and the resistivity.

${ }^{10}$ We thank Bill Matthaeus for pointing out this reference to us.
} 
a) $t=0$

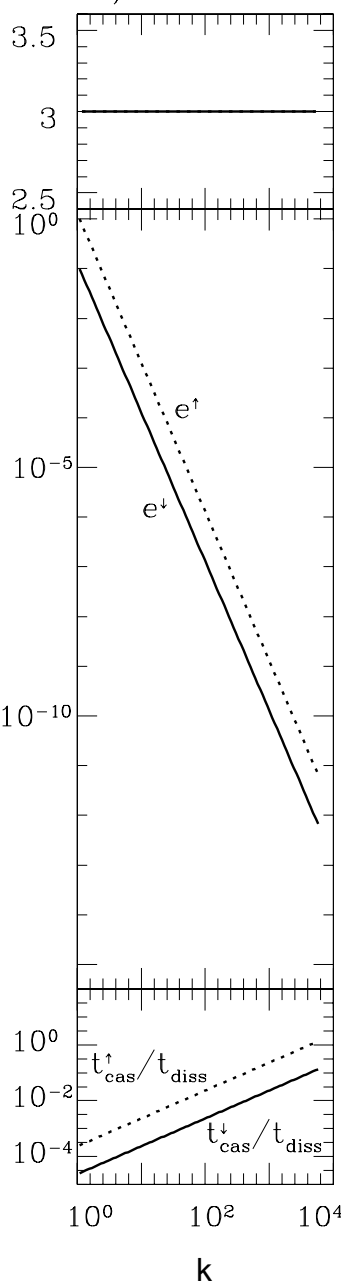

b) $t=0.005$

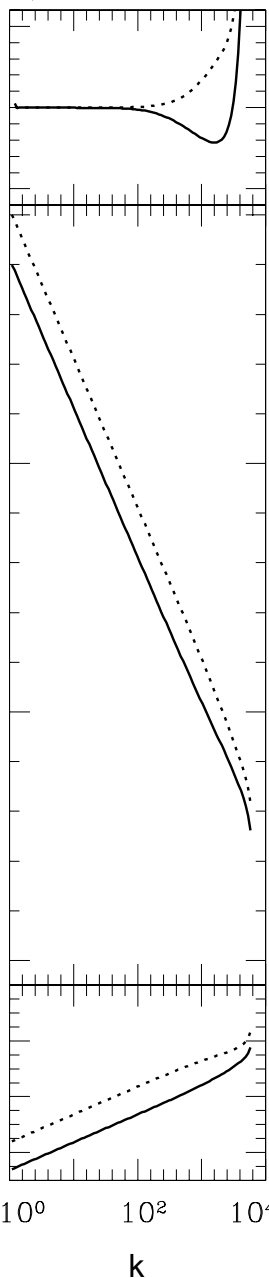

c) $t=0.5$
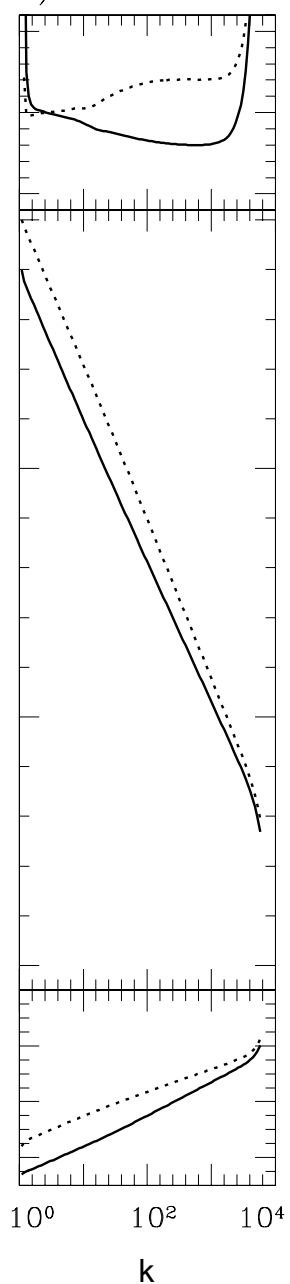

d) $t=50$

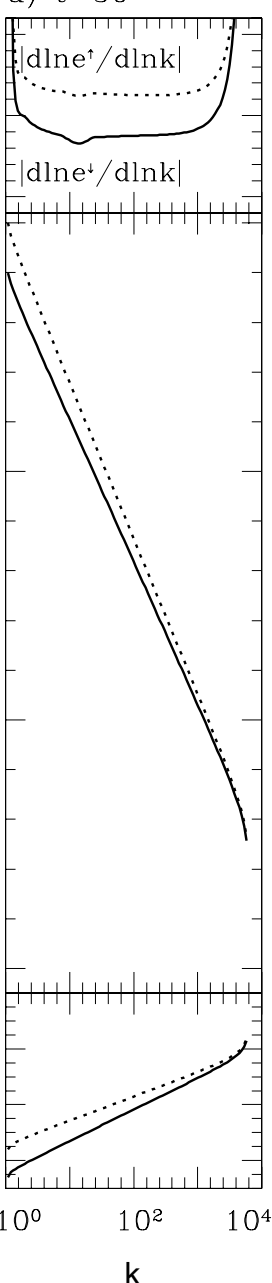

Fig. 1.-Simulation of kinetic equations with fixed energies at the outer scale. (a) Initial condition; $(b-d)$ output of code at three times. Within each panel, the middle segment shows the spectra of $e^{\uparrow}$ (dotted line) and $e^{\downarrow}$ (solid line); the top segment shows their derivatives, $\left|d \ln e^{\uparrow} / d \ln k\right|$ and $\left|d \ln e^{\downarrow} / d \ln k\right|$, and the bottom segment shows the ratio of their cascade times to the dissipation time, $t_{\text {cas }}^{\dagger} / t_{\text {diss }} \sim \nu /\left(k^{2} e^{\downarrow}\right)$ and $t_{\text {cas }}^{\downarrow} / t_{\text {diss }} \sim \nu /\left(k^{2} e^{\dagger}\right)$ (eqs. [57] and [59]). See $\S 6.2$ for an interpretation of the results.

satisfy $w_{\lambda}^{\uparrow} w_{\lambda}^{\downarrow} \propto \lambda$ (eq. [19]). These two conditions completely characterize the steady-state spectra, as long as $w_{\lambda}^{\uparrow}$ and $w_{\lambda}^{\lambda}$ are specified at the outer scale. It is a remarkable feature of weak MHD turbulence that the dynamics at the dissipation scale dramatically affect the entire cascade. Normally, the energy in turbulent cascades is viewed as flowing unimpeded from larger scales to smaller scales. Energy is injected at the outer scale and swallowed up at the dissipation scale. But in weak MHD turbulence, if initially the spectra are not pinned, then the spectrum with lower energy becomes backed up, and its energy increases until pinning occurs.

In the remainder of this section, we derive the scalings in steady state. We denote the dissipation scale by $\lambda_{\text {diss }}$, and the value of $w_{\lambda}^{\uparrow}$ and $w_{\lambda}^{\downarrow}$ at $\lambda_{\text {diss }}$ by $w_{\text {diss. }}$. We can then express the energy spectra as

$$
\begin{aligned}
& w_{\lambda}^{\uparrow}=w_{\text {diss }}\left(\frac{\lambda}{\lambda_{\text {diss }}}\right)^{(1+\alpha) / 2}, \\
& w_{\lambda}^{\downarrow}=w_{\text {diss }}\left(\frac{\lambda}{\lambda_{\text {diss }}}\right)^{(1-\alpha) / 2} .
\end{aligned}
$$

These spectra are valid in the "inertial range," i.e., on length scales larger than the dissipation scale and smaller than the outer scale. There are three parameters that must be calculated to constrain the spectra: $w_{\text {diss }}, \lambda_{\text {diss }}$, and $\alpha$.

For definiteness, we assume that dissipation is caused by a diffusive process, described by a viscous term of the form $\nu \boldsymbol{\nabla}^{2} \boldsymbol{v}$ in equation (1) and a resistive term of the form $\nu \boldsymbol{\nabla}^{2} \boldsymbol{B}$ in equation (2). This implies that the magnetic Prandtl number is equal to $1 .{ }^{11}$ The dissipative timescale is

$$
t_{\text {diss }}(\lambda) \simeq \frac{\lambda^{2}}{\nu}
$$

At the dissipation scale, the cascade time is equal to $t_{\text {diss }}$, i.e., $t_{\text {cas }}\left(\lambda_{\text {diss }}\right)=t_{\text {diss }}\left(\lambda_{\text {diss }}\right)$, which implies that

$$
\frac{\lambda_{\text {diss }}^{2}}{\nu} \simeq\left(\frac{\lambda_{\text {diss }}}{w_{\text {diss }}}\right)^{2} \frac{v_{\mathrm{A}}}{\Lambda},
$$

after using equations (16) and (24); so

$$
w_{\text {diss }} \simeq\left(\frac{\nu v_{\mathrm{A}}}{\Lambda}\right)^{1 / 2} \text {. }
$$

\footnotetext{
${ }^{11}$ It is straightforward to consider other forms for the dissipation. We consider magnetic Prandtl numbers greater than unity in $\S 8$, and hyperviscosity in $\S 9$.
} 
Thus, when the dissipation is caused by a diffusive process, $w_{\text {diss }}$ is independent of the outer-scale energies and the inertial-range fluxes. This is not true for $\lambda_{\text {diss }}$ or $\alpha$.

To calculate $\lambda_{\text {diss }}$ and $\alpha$, we consider two alternative scenarios: specified energies at the outer scale and specified fluxes.

\subsection{Fixed Energies at the Outer Scale}

Suppose that the energies are specified at the outer scale, $\lambda_{\text {out }}$, where $w_{\lambda}^{\uparrow}$ and $w_{\lambda}^{\downarrow}$ are denoted by $w_{\lambda_{\text {out }}}^{\uparrow}$ and $w_{\lambda_{\text {out }}}^{\downarrow}$. Since $w_{\lambda}^{\uparrow} w_{\lambda}^{\downarrow} / \lambda=w_{\text {diss }}^{2} / \lambda_{\text {diss }}$,

$$
\lambda_{\text {diss }} \simeq \frac{\lambda_{\text {out }}}{w_{\lambda_{\text {out }}^{\uparrow}}^{\uparrow} w_{\lambda_{\text {out }}}^{\downarrow}} \frac{\nu v_{\mathrm{A}}}{\Lambda},
$$

after using equation (26).

Dividing equation (22) by equation (23) yields $w_{\lambda_{\text {out }}}^{\uparrow} / w_{\lambda_{\text {out }}}^{\downarrow}=\left(\lambda_{\text {out }} / \lambda_{\text {diss }}\right)^{\alpha}$, so

$$
\alpha=\frac{\ln \left(w_{\lambda_{\text {out }}}^{\uparrow} / w_{\lambda_{\text {out }}}^{\downarrow}\right)}{\ln \left(\lambda_{\text {out }} / \lambda_{\text {diss }}\right)} \simeq \frac{\ln \left(w_{\lambda_{\text {out }}}^{\uparrow} / w_{\lambda_{\text {out }}}^{\downarrow}\right)}{\ln \left[w_{\lambda_{\text {out }}}^{\downarrow} w_{\lambda_{\text {out }}}^{\downarrow} \Lambda /\left(\nu v_{\mathrm{A}}\right)\right]} .
$$

With $w_{\lambda_{\text {out }}}^{\uparrow} / w_{\lambda_{\text {out }}}^{\downarrow}$ fixed, the cascade is balanced $(\alpha \rightarrow 0)$ in the limit that the inertial range is infinitely large $\left(\lambda_{\text {out }} / \lambda_{\text {diss }} \rightarrow \infty\right)$.

Inserting equations (26), (27), and (28) into the spectra (eqs. [22] and [23]) gives the solution to the steady-state imbalanced weak cascade, assuming that $w_{\lambda_{\text {out }}}^{\uparrow}$ and $w_{\lambda_{\text {out }}}^{\downarrow}$ are specified.

Although we have solved for the spectra, recall from $\S 3.2$ that heuristic arguments are insufficient for calculating the ratio of the fluxes that are carried by these spectra, $\epsilon^{\uparrow} / \epsilon^{\downarrow}$. In the following subsection, we show how $\epsilon^{\uparrow} / \epsilon^{\downarrow}$ is related to the spectra. This relation is particularly important for solving the inverse problem: given the fluxes $\epsilon^{\uparrow}$ and $\epsilon^{\downarrow}$, what are the spectra of $w_{\lambda}^{\uparrow}$ and $w_{\lambda}^{\downarrow}$ ?

\subsection{Fixed Fluxes}

Physically, we expect that when the fluxes are fixed, the cascade should be completely constrained. In this subsection, we solve for the spectra given $\epsilon^{\uparrow}$ and $\epsilon^{\downarrow}$. To accomplish this, the dimensionless coefficients of equations (18) and (20) are required. For a given power-law solution, $w_{\lambda}^{\uparrow} \propto \lambda^{(1+\alpha) / 2}$ and $w_{\lambda}^{\downarrow} \propto \lambda^{(1-\alpha) / 2}$, these coefficients depend on $\alpha$ :

$$
\begin{aligned}
\epsilon^{\uparrow} & =f(\alpha)\left(\frac{w_{\lambda}^{\uparrow} w_{\lambda}^{\downarrow}}{\lambda}\right)^{2} \frac{\Lambda}{v_{\mathrm{A}}}, \\
\epsilon^{\downarrow} & =f(-\alpha)\left(\frac{w_{\lambda}^{\uparrow} w_{\lambda}^{\downarrow}}{\lambda}\right)^{2} \frac{\Lambda}{v_{\mathrm{A}}},
\end{aligned}
$$

where $f(\alpha)$ is a dimensionless function of $\alpha$ that must be calculated. By the symmetry between up and down waves, $\epsilon^{\uparrow}$ and $\epsilon^{\downarrow}$ are both proportional to the same function $f$, evaluated at $\pm \alpha$. Since heuristic arguments are insufficient to calculate the function $f$, it is fortunate that weak turbulence can be analyzed with perturbation theory. Galtier et al. (2000) computed $f$. We compute it in equation (A29) in the Appendix.
The ratio of the fluxes is related to $\alpha$ by

$$
\frac{\epsilon^{\uparrow}}{\epsilon^{\downarrow}}=\frac{f(\alpha)}{f(-\alpha)} .
$$

The limit $|\alpha| \ll 1$ is particularly interesting. For given outerscale energies, if the inertial range is very large, then the steady-state cascade is nearly balanced (see the discussion below eq. [28]), and $|\alpha| \ll 1$. In this limit, we show in the Appendix that $f(\alpha) \simeq f(0)(1+0.5 \alpha)$ (see eq. [A30]). Thus,

$$
\frac{\epsilon^{\uparrow}}{\epsilon^{\downarrow}}-1 \simeq \alpha, \quad|\alpha| \ll 1 .
$$

To linear order in $\alpha$, the product of the fluxes is independent of $\alpha$ :

$$
\begin{aligned}
\epsilon^{\uparrow} \epsilon^{\downarrow} & \simeq[f(0)]^{2}\left(\frac{w_{\lambda}^{\uparrow} w_{\lambda}^{\downarrow}}{\lambda}\right)^{4}\left(\frac{\Lambda}{v_{\mathrm{A}}}\right)^{2} \\
& =[f(0)]^{2}\left(\frac{w_{\text {diss }}^{2}}{\lambda_{\text {diss }}}\right)^{4}\left(\frac{\Lambda}{v_{\mathrm{A}}}\right)^{2}, \quad|\alpha| \ll 1 .
\end{aligned}
$$

In the Appendix, we show that $f(0)=1.87$ (eq. A31]). Although for most purposes the precise value of $f(0)$ is unimportant, we shall need it when discussing our numerical simulations.

To summarize, in the limit of small $|\alpha|$, if $\epsilon^{\uparrow}$ and $\epsilon^{\downarrow}$ are specified, then the spectra of $w_{\lambda}^{\uparrow}$ and $w_{\lambda}^{\downarrow}$ are given by equations (22) and (23), with $w_{\text {diss }}, \alpha$, and $\lambda_{\text {diss }}$ given by equations (26), (32), and (33). Note that, to first order in $\alpha$, the only relation that depends on the kinetic equation is equation (32). ${ }^{12}$

If, instead of specifying $\epsilon^{\uparrow}$ and $\epsilon^{\downarrow}$, we specify the outer scale energies-in which case the spectra are given in $\S 4.1-$ then equations (32) and (33) give the resulting fluxes.

\subsection{Three Peripheral Issues}

This subsection may be skipped on a first reading, as it does not impact our main line of argument.

\subsubsection{Locality}

In $\S 3.1$, it is assumed that the dominant interactions are those between wavepackets that have comparable transverse length scales, i.e., interactions are "local" in length scale. In this section, we justify this assumption.

We focus on the cascading of an upgoing wavepacket by downgoing ones. Let the upgoing wavepacket have transverse size $\lambda$, and let the downgoing wavepackets each have transverse size $l$, parallel size $\Lambda$, and amplitude $w_{l}^{\downarrow}$. The upgoing wavepacket cascades when the field lines it is following wander a distance comparable to its transverse size, $\lambda$.

We consider first the case of $l<\lambda$. Two field lines that are separated by $\lambda$ at the head of the downgoing wavepackets wander independently of each other. Their transverse separation after $N$ downgoing wavepackets increases by

$$
N^{1 / 2}\left(w_{l}^{\downarrow} / v_{\mathrm{A}}\right) \Lambda, \quad l<\lambda .
$$

Conversely, if $l>\lambda$, then the magnetic field can be expanded to linear order in $\lambda$, so the magnetic field at two

\footnotetext{
12 Aside from the uninteresting dependence of eq. (33) on $f(0)$.
} 
points separated by $\lambda$ differs by $\sim w_{l}^{\downarrow} \lambda / l$. Consequently, the field lines separate by

$$
N^{1 / 2}\left(w_{l}^{\downarrow} / v_{\mathrm{A}}\right)(\lambda / l) \Lambda, \quad l>\lambda,
$$

so long as this separation is smaller than $\lambda$.

For interactions to be local, i.e., for the amount of field line wander seen by an up-wave of transverse size $\lambda$ to be maximized by those down-waves that have $l \sim \lambda$, the following two conditions must hold: (1) $w_{l}^{\downarrow}$ is an increasing function of $l$ (eq. [34]); and (2) $w_{l}^{\downarrow} / l$ is a decreasing function of $l$ (eq. [35]). So the cascade is local if

$$
0<\frac{d \ln w_{\lambda}^{\downarrow}}{d \ln \lambda}<1 .
$$

The same condition clearly holds for $w_{\lambda}^{\uparrow}$. In terms of the steady-state scalings, $w_{\lambda}^{\uparrow} \propto \lambda^{(1+\alpha) / 2}$ and $w_{\lambda}^{\downarrow} \propto \lambda^{(1-\alpha) / 2}$, the condition

$$
-1<\alpha<1
$$

is required for the cascade to be local; otherwise, nonlocal effects are important. Galtier et al. (2000) derived the inequalities in relation (37) from their kinetic equation.

There is a second reason why the condition $d \ln w_{\lambda}^{\uparrow} / d \ln \lambda<1$ is necessary for our heuristic arguments to be valid. Consider a single Fourier mode with wavelength $l$ and amplitude $w_{l}^{\uparrow}$; then the typical difference in $w^{\uparrow}$ between two points separated by $\lambda<l$ is $\sim w_{l}^{\uparrow} \lambda / l$, expanding to linear order in $\lambda$. So, if the condition $d \ln w_{\lambda}^{\uparrow} / d \ln \lambda<1$ is violated, then the contribution to $w_{\lambda}^{\uparrow}$ (i.e., to the typical difference in $w^{\uparrow}$ between two points separated by $\lambda$ ) is dominated by those Fourier modes that have wavelengths $l \gg \lambda$. This is a different kind of nonlocality than considered previously: the upgoing energy that crosses length scale $\lambda$ comes directly from much larger scales (with $l \gg \lambda$ ).

\subsubsection{Transition to Strong Turbulence}

Weak turbulence is applicable when $w_{\lambda}^{\downarrow} \Lambda / v_{\mathrm{A}} \ll \lambda$ and $w_{\lambda}^{\uparrow} \Lambda / v_{\text {A }} \ll \lambda$ (eq. [15]). Since $\lambda$ decreases faster than both $w_{\lambda}^{\downarrow}$ and $w_{\lambda}^{\uparrow}$ (eq. [36]), even if these inequalities are satisfied at large length scales, they are violated at small ones. Thus, weak turbulence has a limited inertial range.

Strong turbulence, which is applicable when the above inequalities are violated, is of greater relevance than weak turbulence for describing astrophysical sites such as the solar wind. In strong turbulence the change in field line separation within a single wavepacket is not smaller than the transverse size of the wavepacket. This has two implications. First, equation (16) for the cascade time is no longer valid; and second, the parallel size of wavepackets $\Lambda$ decreases toward smaller scales because the head and tail of a wavepacket are independently cascaded. The balanced strong cascade is worked out by Goldreich \& Sridhar (1995); we discuss the imbalanced strong cascade in a future paper. Strong turbulence is more difficult to analyze than weak turbulence because it does not submit to perturbation theory. Nonetheless, a number of the features of weak turbulence that we develop in the present paper are applicable to strong turbulence. This is one of our motivations for studying the weak cascade.

Since this paper is concerned with weak turbulence, we choose the dissipation scale to be sufficiently large that the entire cascade is weak, i.e., we require that

$$
\frac{w_{\lambda_{\text {diss }}}^{\uparrow} \Lambda}{v_{\mathrm{A}} \lambda_{\text {diss }}} \ll 1
$$

and similarly for $w_{\lambda_{\text {diss }}}^{\downarrow}$. Using equations (26) and (27), we can rewrite this condition as

$$
\frac{w_{\lambda_{\text {out }}}^{\uparrow} w_{\lambda_{\text {out }}}^{\downarrow}}{v_{\mathrm{A}}^{2}} \ll \frac{\lambda_{\text {out }}}{\Lambda}\left(\frac{\nu}{\Lambda v_{\mathrm{A}}}\right)^{1 / 2} .
$$

There is also a lower limit on the product of the outerscale energies, set by the requirement that the dissipation scale be smaller than the outer scale, which implies that

$$
\frac{w_{\lambda_{\text {out }}}^{\uparrow} w_{\lambda_{\text {out }}}^{\downarrow}}{v_{\mathrm{A}}^{2}} \gg \frac{\nu}{\Lambda v_{\mathrm{A}}},
$$

(see eq. [27]). Typically, one expects that $\Lambda \sim \lambda_{\text {out }}$; so as long as $\nu \ll \Lambda v_{\mathrm{A}}$, the above two inequalities can be satisfied with appropriately chosen $w_{\lambda_{\text {out }}}^{\uparrow} w_{\lambda_{\text {out }}}^{\downarrow}$.

\subsubsection{The Steady-State Spectra of $w_{z}^{\uparrow}$ and $w$ and of a Passive Scalar}

In $\S 2$ we showed that the parallel components $w_{z}^{\uparrow}$ and $w_{z}^{\downarrow}$ do not affect the evolution of the perpendicular components, $\boldsymbol{w}^{\uparrow}$ and $\boldsymbol{w}^{\downarrow} .^{13}$ By contrast, the perpendicular components control the evolution of the parallel ones.

The steady-state spectra of the parallel components can be derived as follows. By analogy between the equation for $w_{z}^{\uparrow}$ (eq. [12]) and that for $\boldsymbol{w}^{\uparrow}$ (eq. [8]), the cascade time of $w_{z}^{\uparrow}$ is similar to that of $\boldsymbol{w}^{\uparrow}$, i.e., it is given by $t_{\text {cas }}^{\uparrow}$ (see eq. [16]). We denote the typical amplitude of the parallel component of the up-wave on length scale $\lambda$ by $w_{z, \lambda}^{\uparrow}$. The cascade rate of the energy of the parallel component is $\left(w_{z, \lambda}^{\uparrow}\right)^{2} / t_{\text {cas }}^{\uparrow}$; in steady state it must be independent of $\lambda$. Comparing this to the cascade rate of the perpendicular component, $\left(w_{\lambda}^{\uparrow}\right)^{2} / t_{\text {cas }}^{\uparrow}$, which must also be independent of $\lambda$, we deduce

$$
w_{z, \lambda}^{\uparrow} \propto w_{\lambda}^{\uparrow} .
$$

Note that the evolution equation for $w_{z}^{\uparrow}$ (eq. [12]) is linear. So the overall amplitude of the $w_{z, \lambda}^{\uparrow}$ spectrum is arbitrary; more accurately, it is set by the value of $w_{z, \lambda}^{\uparrow}$ at the outer scale. Of course, a similar relation holds for the downwaves:

$$
w_{z, \lambda}^{\downarrow} \propto w_{\lambda}^{\downarrow} .
$$

Finally, we consider the spectrum of a passive scalar, $s$, whose evolution is described by equation (14). From this equation, it is apparent that the cascade time of the passive scalar is given by either $t_{\text {cas }}^{\uparrow}$ or $t_{\text {cas }}^{\downarrow}$, whichever is shorter. So, by the same reasoning that we used in deriving equation (41), we deduce

$$
s_{\lambda} \propto \min \left(w_{\lambda}^{\uparrow}, w_{\lambda}^{\downarrow}\right),
$$

where $s_{\lambda}$ is the typical value of $s$ on length scale $\lambda$. Because of the pinning of the spectra, the lesser of the two spectra $w_{\lambda}^{\uparrow}$ and $w_{\lambda}^{\downarrow}$ is also the flatter. So $s_{\lambda}$ is proportional to the flatter of $w_{\lambda}^{\uparrow}$ and $w_{\lambda}^{\downarrow}$.

\footnotetext{
13 Recall that we drop the $\perp$ label.
} 


\section{KINETIC EQUATIONS IN WEAK TURBULENCE}

Weak turbulence can be analyzed with perturbation theory. As a consequence, the evolution of the energy spectra of the up- and down-waves is described by a closed set of two equations; in other words, the two-point correlation functions evolve independently of all higher order correlation functions. This is a great simplification.

Evolution equations for the energy spectra - the " kinetic equations"-were obtained in Galtier et al. (2000, 2002). We present an alternate, more physical, derivation in the Appendix. Such a derivation is useful because it clears up a number of erroneous claims that have been made in the literature - in particular, the claim of Goldreich \& Sridhar (1997) that perturbation theory is inapplicable.

In the following, we summarize the result derived in the Appendix. The kinetic equations are given in Fourier space. We Fourier transform $\boldsymbol{w}^{\uparrow}(x, y, z, t)$ and $\boldsymbol{w}^{\downarrow}(x, y, z, t)$ in $x$ and $y$ (but not $z$ ), and denote the transforms by $\boldsymbol{w}_{k}^{\uparrow}(z, t)$ and $\boldsymbol{w}_{\boldsymbol{k}}^{\downarrow}(z, t)$, where $\boldsymbol{k}$ is purely transverse $\left(k_{z} \equiv 0\right)$. We define the energy spectra $e^{\uparrow}$ and $e \downarrow$ such that

$$
\begin{aligned}
& \left\langle\boldsymbol{w}_{\boldsymbol{k}}^{\uparrow}(z, t) \cdot \boldsymbol{w}_{\boldsymbol{k}^{\prime}}^{\uparrow}(z, t)\right\rangle=e^{\uparrow}(k, t) \delta\left(\boldsymbol{k}+\boldsymbol{k}^{\prime}\right) \\
& \left\langle\boldsymbol{w}_{\boldsymbol{k}}^{\downarrow}(z, t) \cdot \boldsymbol{w}_{\boldsymbol{k}^{\prime}}^{\downarrow}(z, t)\right\rangle=e^{\downarrow}(k, t) \delta\left(\boldsymbol{k}+\boldsymbol{k}^{\prime}\right),
\end{aligned}
$$

where $\delta(\boldsymbol{k})$ is a two-dimensional Dirac delta function, and angle brackets denote both an ensemble average and an average over $z$. (We assume that the turbulence is homogeneous in $z$.) Both $e^{\uparrow}$ and $e^{\downarrow}$ are real. The turbulence is isotropic in the transverse plane, so $e^{\uparrow}$ and $e \downarrow$ are functions of the magnitude of $\boldsymbol{k}$.

From equation (A24) in the Appendix, the kinetic equation for the up-waves is

$$
\begin{aligned}
\left.\frac{\partial}{\partial t}\right|_{k} e^{\uparrow}(k, t)= & \frac{\Lambda}{v_{\mathrm{A}}} k^{2} \int_{0}^{\infty} d k_{2} \int_{0}^{2 \pi} d \theta k_{2}^{3}\left[e^{\uparrow}\left(k_{2}, t\right)-e^{\uparrow}(k, t)\right] \\
& \times \sin ^{2} \theta \cos ^{2} \theta \frac{e^{\downarrow}\left(k_{1}, t\right)}{k_{1}^{2}}-\nu k^{2} e^{\uparrow}(k, t),
\end{aligned}
$$

where

$$
k_{1} \equiv\left(k^{2}+k_{2}^{2}-2 k k_{2} \cos \theta\right)^{1 / 2} .
$$

We have included a term describing diffusive dissipation on small length scales, $-\nu k^{2} e^{\uparrow}$, where $\nu$ is the viscosity, which is assumed to be equal to the resistivity.

Because of the symmetry between up- and down-waves, the equation for $e \downarrow$ is the same as that for $e^{\uparrow}$, but with $\uparrow$ and $\downarrow$ everywhere switched. The steady-state relation between the energy and the flux that we use above (eq. [29]) is obtained in the Appendix by setting equation (46) to zero.

Within an order-unity factor, $k^{2} e^{\uparrow} \sim\left(w_{\lambda}^{\uparrow}\right)^{2}$ when $\lambda=1 / k$. Recall from $\S 3$ that $w_{\lambda}^{\uparrow}$ is the "typical "value of $\boldsymbol{w}^{\uparrow}$ on length scale $\lambda$; more precisely, it can be defined as the square root of the second-order structure function of $\boldsymbol{w}^{\uparrow}$. Therefore, the steady-state scaling $w_{\lambda}^{\uparrow} \propto \lambda^{(1+\alpha) / 2}$ (eq. [22]) corresponds to $e^{\uparrow} \propto k^{-(3+\alpha)}$; similarly, $w_{\lambda}^{\downarrow} \propto \lambda^{(1-\alpha) / 2}$ corresponds to $e^{\downarrow} \propto k^{-(3-\alpha)}$.

\subsection{Energy and Flux in the Kinetic Equation}

The energy per unit mass in up-waves is $\left\langle\left|\boldsymbol{w}^{\uparrow}\right|^{2}\right\rangle / 2=$ $\frac{1}{2}(2 \pi)^{-4} \int e^{\uparrow} d^{2} \boldsymbol{k}$, so $e^{\uparrow}$ is proportional to the energy per unit $d^{2} \boldsymbol{k}$. Conservation of up-wave energy implies that $(d / d t) \int\left(k e^{\uparrow}\right) d k=0$, assuming isotropy and neglecting dissipation. This can be seen immediately from equation (46) if we rewrite it (without the diffusive term) as

$$
(\partial / \partial t)\left(k e_{k}^{\uparrow}\right)=\left(\Lambda / v_{\mathrm{A}}\right) \int_{0}^{\infty}\left(e_{k_{2}}^{\uparrow}-e_{k}^{\uparrow}\right) S^{\downarrow}\left(k, k_{2}\right) d k_{2},
$$

since $S \downarrow$ is symmetric,

$S^{\downarrow}\left(k, k_{2}\right) \equiv\left(k k_{2}\right)^{3} \int_{0}^{2 \pi} \sin ^{2} \theta \cos ^{2} \theta e_{k_{1}}^{\downarrow} k_{1}^{-2}=S^{\downarrow}\left(k_{2}, k\right)$,

and $e_{2}^{\uparrow}-e_{k}^{\uparrow}$ is antisymmetric. In comparing our results with those of Galtier et al. (2000), note that these authors use the energy per unit $k$, which they denote $E^{+}$, i.e., $E^{+} \sim k e^{\uparrow}$, within a multiplicative constant.

The energy flux $\epsilon^{\uparrow}$ is the net rate at which energy flows across a given wavenumber $k$. We define it as the integral of the right-hand side of equation (46) over $d^{2} \boldsymbol{k} /(2 \pi)$, from some particular $k$ to infinity, so that the kinetic equation can be written as

$$
(\partial / \partial t)\left(k e_{k}^{\uparrow}\right)=-(\partial / \partial k) \epsilon^{\uparrow} .
$$

Explicitly, we can write the flux in the form

$$
\epsilon^{\uparrow}=\int_{0}^{k} d p \int_{k}^{\infty} d q\left(e_{p}^{\uparrow}-e_{q}^{\uparrow}\right) S^{\downarrow}(p, q) .
$$

It is trivial to verify that $\partial / \partial k$ of this expression is equal to minus the right-hand side of equation (46). The positive term in equation $(51)$ is the rate at which energy flows from wavenumbers smaller than $k$ to those greater than $k$; the negative term is similar, but with the origin and destination of the energy switched.

\section{NUMERICAL SIMULATIONS OF KINETIC EQUATIONS}

In this section we discuss numerical simulations of the kinetic equations that verify our previous heuristic discussions: in particular, the pinning of spectra and the scaling of the spectra in steady state. Galtier et al. (2000) perform numerical simulations of the kinetic equation. However, their investigation of the imbalanced cascade is incomplete. In particular, they only plot spectra of the product $e^{\uparrow} e^{\downarrow}$. They do not discuss the pinning of the spectra at the dissipation scale, which is crucial to the evolution of the cascade.

It is much faster to simulate kinetic equations than the full equations of motion for $\boldsymbol{w}^{\uparrow}$ and $\boldsymbol{w} \downarrow$. There are a number of reasons for this. First, the kinetic equations are only onedimensional, since homogeneity has been assumed parallel to the mean magnetic field, and isotropy has been assumed in the plane transverse to the mean magnetic field. Second, and more importantly, the averaged energies $e^{\uparrow}$ and $e^{\downarrow}$ are much smoother functions of $k$ than $\boldsymbol{w}^{\uparrow}$ and $\boldsymbol{w} \downarrow$. Thus, a logarithmically spaced grid can be used, which greatly reduces the number of variables that need to be evolved. The result is an enormous reduction in computational time. A typical kinetic simulation takes a few hours on a PC to reach steady state. A comparable fully three-dimensional MHD simulation would require many months, if not years, on the fastest supercomputers. 
In the future, we expect that more elaborate simulations than those considered in the present paper will be performed. One straightforward extension is the simulation of the kinetic equations without averaging in the direction parallel to the mean magnetic field. This would require a twodimensional simulation, i.e., $k$ and $z$ would be the independent variables (in addition to the time). The corresponding kinetic equation is nearly identical to the one we simulate in this paper; one must, however, keep track of separate planes transverse to the mean magnetic field. Such a simulation would enable one to investigate the role of fluctuations in the energy spectra amongst different planes. Although we do not expect that such fluctuations play a large role, numerical verification of this is desirable. Three-dimensional simulations of the full MHD equations are even more desirable, though much more difficult. Maron \& Goldreich (2001), Cho \& Vishniac (2000), and Müller \& Biskamp (2000) have performed three-dimensional simulations of strong MHD turbulence. Such simulations stretch the abilities of the fastest supercomputers. Unfortunately, simulations of weak turbulence are even more difficult. This is because each wavepacket must interact with many oppositely directed ones before it cascades; by contrast, in strong MHD turbulence a wavepacket cascades in the time that it crosses a single oppositely directed one. Nonetheless, simulations of weak MHD turbulence are of interest because they could verify (or refute) our present investigation. In addition, they could shed light on the transition from weak to strong turbulence.

\subsection{Computational Setup}

We simulate the kinetic equations (eq. [46] and the corresponding equation for $e^{\downarrow}$ ). Recall that we assume a strong mean magnetic field in the $\hat{z}$ direction, and that wavevectors $\boldsymbol{k}$ are perpendicular to $\hat{\boldsymbol{z}}$. We average the fluctuations both over the $z$-dimension and, for a given $k \equiv|\boldsymbol{k}|$, over orientations of $\boldsymbol{k}$.

We formally set

$$
\frac{\Lambda}{v_{\mathrm{A}}}=\frac{1}{2}
$$

in our numerical simulations of equation (46). ${ }^{14}$ Equation

${ }^{14}$ More precisely, our setting of $\Lambda / v_{\mathrm{A}}=1 / 2$ can be understood as follows. We can write the kinetic equations in terms of the following dimensionless variables:

$$
\tilde{e}^{\uparrow, \downarrow} \equiv \frac{e^{\uparrow, \downarrow}}{e_{\mathrm{typ}}}, \quad \tilde{k} \equiv \frac{k}{k_{\mathrm{typ}}}, \quad \tilde{t} \equiv \frac{2 \Lambda}{v_{\mathrm{A}}} k_{\mathrm{typ}}^{4} e_{\mathrm{typ}} t, \quad \tilde{\nu} \equiv \frac{v_{\mathrm{A}}}{2 \Lambda} \frac{\nu}{k_{\mathrm{typ}}^{2} e_{\mathrm{typ}}},
$$

where $e_{\mathrm{typ}}$ and $k_{\mathrm{typ}}$ denote "typical" values of $e$ and $k$; they are arbitrary constants that factor out of the kinetic equations. The resulting kinetic equation is the same as eq. (46), but with variables replaced by their counterparts with tilde's, and with $\Lambda / v_{\mathrm{A}} \rightarrow 1 / 2$. Thus, it is really the variables with tildes that we simulate, although for clarity we drop the tildes in our exposition. The arbitrary parameters $e_{\mathrm{typ}}$ and $k_{\mathrm{typ}}$ can be used transform the results of our simulations to physical values of the variables. For example, the dimensionless ratio that must be small in weak turbulence (see eq. [15]) is

$$
\frac{w_{\lambda}^{\uparrow} \Lambda}{v_{\mathrm{A}} \lambda} \sim \frac{\Lambda}{v_{\mathrm{A}}} k^{2}\left(e^{\uparrow}\right)^{1 / 2}=\left[k_{\mathrm{typ}}^{2} e_{\mathrm{typ}}^{1 / 2} \Lambda / v_{\mathrm{A}}\right] \tilde{k}^{2}\left(\tilde{e}^{\uparrow}\right)^{1 / 2} .
$$

Note that this ratio can be made as small as desired with appropriately chosen values of $e_{\text {typ }}$ and $k_{\text {typ }}$.
(46) becomes

$$
\begin{aligned}
\left.\frac{\partial}{\partial t}\right|_{k} e^{\uparrow}(k, t)= & k \int_{0}^{\infty} d k_{2} \int_{\left|k-k_{2}\right|}^{\left|k+k_{2}\right|} d k_{1} k_{2}^{2}\left[e^{\uparrow}\left(k_{2}, t\right)-e^{\uparrow}(k, t)\right] \\
& \times \sin \theta \cos ^{2} \theta \frac{e^{\downarrow}\left(k_{1}, t\right)}{k_{1}}-\nu k^{2} e^{\uparrow}(k, t)
\end{aligned}
$$

where $\theta$ is a function of $k_{1} / k$ and $k_{2} / k$ (given in eq. [47]), and we have changed integration variables from $\theta$ to $k_{1}$ in the second integral. Our method of integration of equation (53) is very similar to that of Galtier et al. (2000). We evaluate all functions of $k$ on a fixed, logarithmically spaced grid with $k=2^{i / 8}, i=1, \ldots, 100$. At the outer scale,

$$
k_{\text {out }}=2^{1 / 8}=1.09 \text {, }
$$

and the maximum $k$ is

$$
k_{\max }=2^{100 / 8}=5793 \text {. }
$$

The double integral over $k_{1}$ and $k_{2}$ is performed by summing the values of the integrand evaluated on the $k$-space grid. The factor $\sin \theta \cos ^{2} \theta$ is averaged in the vicinity of each grid point, i.e., at each $\left(k_{1}, k_{2}\right)$. Since $\theta$ is a function of $k_{1} / k$ and $k_{2} / k$, and since the grid is logarithmic, the averaged angular factor can be precomputed and stored in a two-dimensional matrix, each element of which is the average in the vicinity of $\left(k_{1} / k, k_{2} / k\right) .{ }^{15}$ We integrate in time with second-order Runge-Kutta.

For the discussions of the simulations that follow, recall that the energies of the waves at a given length scale $\lambda=1 / k$ are, within unimportant multiplicative constants,

$$
\left(w_{\lambda}^{\uparrow}\right)^{2} \sim k^{2} e^{\uparrow}(k), \quad\left(w_{\lambda}^{\downarrow}\right)^{2} \sim k^{2} e^{\downarrow}(k) .
$$

The cascade times of the up- and downgoing waves are, respectively,

$$
t_{\mathrm{cas}}^{\uparrow}(k) \sim\left[k^{4} e^{\downarrow}(k)\right]^{-1}, \quad t_{\mathrm{cas}}^{\downarrow}(k) \sim\left[k^{4} e^{\uparrow}(k)\right]^{-1}
$$

(see eq. [16]). The dissipation time is

$$
t_{\text {diss }}(k)=\left(\nu k^{2}\right)^{-1}
$$

(see eq. [24]). In the simulations in this section, the viscosity is

$$
\nu=3 \times 10^{-5}
$$

this implies that $t_{\text {diss }}\left(k_{\max }\right)=0.001$.

\footnotetext{
${ }^{15}$ In evaluating the integral near $k_{\text {out }}$, the values of $e^{\uparrow}\left(k_{2}<k_{\text {out }}\right)$ and $e^{\downarrow}\left(k_{1}<k_{\text {out }}\right)$ are required. Since these values are off the grid, some method of extrapolation is needed. In the runs that we present in this paper, we extrapolate with $e^{\uparrow}, e^{\downarrow} \propto k^{-2}$. With this extrapolation, there is no energy transfer from modes with $k<k_{\text {out }}$ to those with $k>k_{\text {out }}$; steeper extrapolations would transfer energy. We have also experimented with a flatter spectrum for $k<k_{\text {out }}: e^{\uparrow}=e^{\dagger}\left(k_{\text {out }}\right), e^{\downarrow}=e^{\downarrow}\left(k_{\text {out }}\right)$. While this changes the behavior near $k \sim k_{\text {out }}$-in particular, it leads to a sharp drop in $e^{\uparrow}$ and $e^{\downarrow}$ between $k=k_{\text {out }}$ and $k=2^{1 / 8} k_{\text {out }}$ by a factor of around 3-the remainder of the spectrum for $k>2^{1 / 8} k_{\text {out }}$ is nearly unaffected. With the $k^{-2}$ extrapolation there is also a drop at $k=k_{\text {out }}$, as can be seen in the top panel of Figure $1 d$, for example; but this drop is much less drastic than with the flat spectrum extrapolation.
} 


\subsection{Fixed Energies at the Outer Scale}

For our first simulation, we fix $e^{\uparrow}\left(k_{\text {out }}\right)=1$ and $e^{\downarrow}\left(k_{\text {out }}\right)=0.1$ throughout the simulation. We do this by adding an injection term at $k=k_{\text {out }}$ to the right-hand side of the kinetic equation (eq. [53]). The two injection terms, $\dot{e}_{\text {inj }}^{\uparrow}$ and $\dot{e}_{\text {ini }}^{\downarrow}$, are adjusted to keep $e^{\uparrow}\left(k_{\text {out }}\right)$ and $e^{\downarrow}\left(k_{\text {out }}\right)$ fixed. At the initial time, we set $e^{\uparrow} \propto k^{-3}$ and $e^{\downarrow} \propto k^{-3}$ (Fig. 1a). These initial spectra are seemingly valid solutions of the steady-state flux relations (eqs. [29] and [30]), with $\alpha=0$ and $\epsilon^{\uparrow}=\epsilon^{\downarrow}$. But the spectra are not pinned. When they are evolved in time, it is seen that they become pinned to each other at the dissipation scale. This pinning happens very quickly - at the dissipation timescale (Fig 1b). The reason for this pinning was discussed in $\S 3.3$, and can be traced in Figures $1 a-1 c$.

The entire $e^{\downarrow}$ spectrum adjusts to $e^{\uparrow}$ on the timescale $t_{\text {cas }}^{\downarrow}\left(k_{\text {out }}\right) \sim 1$. The $e^{\uparrow}$ spectrum takes longer to adjust, since $t_{\text {cas }}^{\text {t }}\left(k_{\text {out }}\right) \sim 10$. By $t=50$, steady state is reached (Fig. $1 d$ ). We discuss the resulting steady-state spectra in $\S 6.4$. We find that, in steady state, the energy injection rates required to keep the outer scale energies fixed are

$$
\dot{e}_{\text {inj }}^{\uparrow}=0.136, \quad \dot{e}_{\text {inj }}^{\downarrow}=0.114 ;
$$

these values are used in our second simulation.

\subsection{Fixed Fluxes}

In our second simulation, we inject energy at fixed rates at $k=k_{\text {out }}$, allowing $e^{\uparrow}\left(k_{\text {out }}\right)$ and $e^{\downarrow}\left(k_{\text {out }}\right)$ free to evolve. We use the same injection rates as we found in steady state in the previous simulation (eq. [60]). We add to $e^{\uparrow}\left(k_{\text {out }}\right)$ and $e^{\downarrow}\left(k_{\text {out }}\right)$ at these fixed rates throughout the present simulation. Since we add to $e^{\uparrow}\left(k_{\text {out }}\right)$ and $e^{\downarrow}\left(k_{\text {out }}\right)$ at the same rate as we did in steady state in the previous simulation, we expect that our second simulation will reach the same steady state as did the first one. We will show that it does. Note that, strictly speaking, $\dot{e}_{\text {inj }}^{\uparrow}$ and $\dot{e}_{\text {inj }}^{\downarrow}$ are not precisely equal to the fluxes. Nonetheless, we ignore this subtlety and refer to the present simulation as one of constant fluxes. We evaluate the fluxes more precisely below.

For our initial condition, we use the spectra obtained in steady state in the first simulation, but multiplied by a constant; specifically, $e^{\uparrow}(k) \rightarrow e^{\uparrow}(k) / 10$ and $e^{\downarrow}(k) \rightarrow e^{\downarrow}(k) \times 10$ (see Fig. 2a). With these initial spectra, and with the aforementioned fluxes, the flux relations (eqs. [29] and [30]) are satisfied - since they were satisfied before the multiplication and division by 10 . But, once again, this is not a valid steady-state solution because the spectra are not pinned at the dissipation scale. When the spectra are evolved in time, the spectra are first pinned (Figs. $2 a-2 c$ ). In this simulation, a) $t=0$

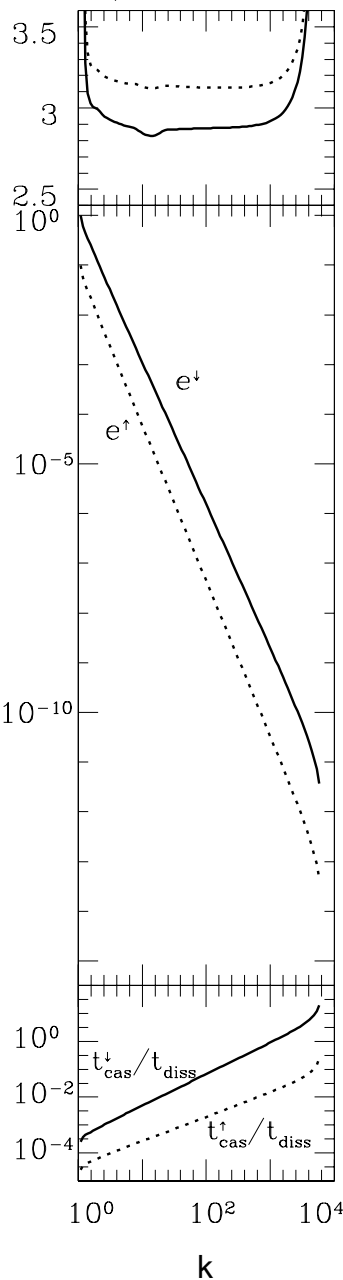

b) $t=0.01$

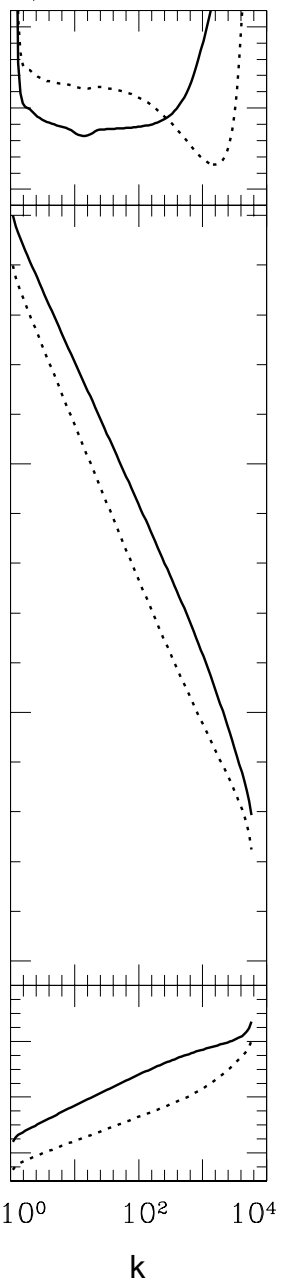

c) $t=0.1$

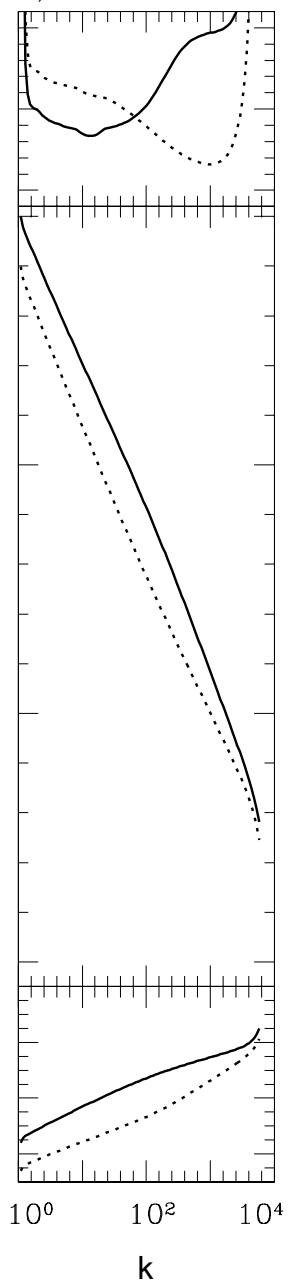

d) $t=250$

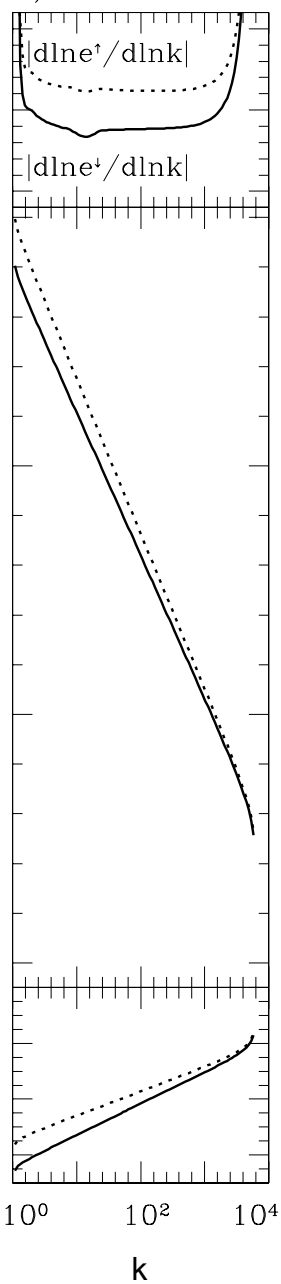

FIG. 2.-Simulation of kinetic equations with fixed fluxes 
the spectra are initially pinned where $t_{\text {cas }}^{\downarrow} \sim t_{\text {diss }}$, which is at $k \sim 1000$. Therefore, the pinning timescale is $\sim t_{\text {diss }}(k=1000) \sim 0.03$.

As expected, the same steady state is reached as in the first simulation (Fig. 2d). In reaching steady state, the two spectra must cross. This happens at $t \sim 40$, when the two spectra are nearly identical, with logarithmic slopes equal to -3 , and with outer scale energy equal to $\sim 0.3$. The time to reach steady state is considerably longer in the constant-flux simulation than in the constant-energy one. Steady state is reached at $t \sim 250$, as opposed to $t \sim 50$ in the previous simulation.

\subsection{Energies and Fluxes in Steady State}

We can quantitatively compare the behavior in steady state with our calculations in $\S 4$. We consider the steady state reached in the two simulations discussed above. Recall that both simulations reach the same steady state, at which point the spectra are as shown in Figures $1 d$ and $2 d$. For our comparisons, we need the following quantities: $\nu=3 \times 10^{-5}$ (eq. [59]), $k_{\text {out }}=1.09$ (eq. [54]), $\Lambda / v_{\mathrm{A}}=1 / 2$ (eq. [52]), $e^{\uparrow}\left(k_{\text {out }}\right)=0.63$, and $e \downarrow\left(k_{\text {out }}\right)=0.066$. For our first simulation, we actually fixed $e^{\uparrow}\left(k_{\text {out }}\right)=1$ and $e^{\downarrow}\left(k_{\text {out }}\right)=0.1$. However, as we discuss in footnote 15 , there is a drop in energy between the first and second grid point. The values $e^{\uparrow}\left(k_{\text {out }}\right)=0.63$, and $e^{\downarrow}\left(k_{\text {out }}\right)=0.066$ are found by taking the power laws seen in steady state for $e^{\uparrow}\left(k>k_{\text {out }}\right)$ and $e^{\downarrow}\left(k>k_{\text {out }}\right)$, and extrapolating them to $k=k_{\text {out }}$.

From equation (27), the dissipation wavenumber is

$$
k_{\text {diss }}=\frac{\Lambda}{\nu v_{\text {A }}}\left[k_{\text {out }}^{6} e^{\dagger}\left(k_{\text {out }}\right) e^{\downarrow}\left(k_{\text {out }}\right)\right]^{1 / 2}=4400,
$$

in agreement with the value seen in Figure $1 d$.

Equation (28) gives

$$
\alpha=\frac{\ln \left[e^{\uparrow}\left(k_{\text {out }}\right) / e^{\downarrow}\left(k_{\text {out }}\right)\right]}{\ln \left[k_{\text {out }}^{4} e^{\uparrow}\left(k_{\text {out }}\right) e^{\downarrow}\left(k_{\text {out }}\right)\left(\Lambda / \nu v_{\mathrm{A}}\right)^{2}\right]}=0.14,
$$

which implies that the steady-state slopes should be

$$
\begin{aligned}
& -\frac{d \ln e^{\uparrow}}{d \ln k}=3+\alpha=3.14, \\
& -\frac{d \ln e^{\downarrow}}{d \ln k}=3-\alpha=2.86 .
\end{aligned}
$$

This is in agreement with the top panel of Figure $1 d$, which shows that the logarithmic slope of $e^{\uparrow}$ in the inertial range, while varying slightly with $k$, mostly remains between 3.1 and 3.15; the logarithmic slope of $e^{\uparrow}$ is between 2.85 and 2.9.

To derive the steady-state formulae that we have used thus far in this section, heuristic arguments suffice. However, to relate energy spectra to fluxes, the kinetic equation is required. From equation (33), we should have

$$
\epsilon^{\uparrow} \epsilon^{\downarrow}=\left[1.87 k_{\text {out }}^{6} e^{\uparrow}\left(k_{\text {out }}\right) e^{\downarrow}\left(k_{\text {out }}\right)\left(\Lambda / v_{\mathrm{A}}\right)\right]^{2}=0.0043,
$$

where the numerical factor 1.87 is obtained from the kinetic equation. A more important application of the kinetic equation is to derive the ratio of the fluxes, for which heuristic arguments are useless. From equation (32), we should have

$$
\epsilon^{\uparrow} / \epsilon^{\downarrow}-1=\alpha=0.14,
$$

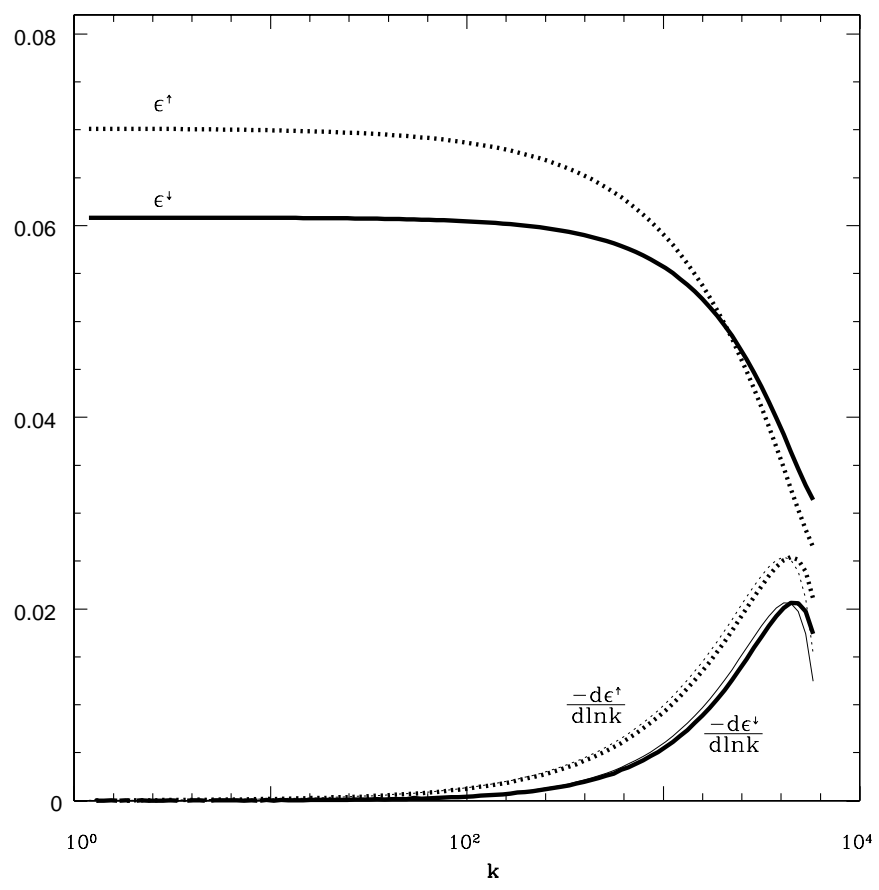

FIG. 3.-Steady-state fluxes and flux gradients. Fluxes and flux gradients are shown as bold lines. Also shown as unlabeled thin lines that nearly overlap the flux gradients are $\nu k^{4} e^{\uparrow}$ (thin dotted line) and $\nu k^{4} e^{\downarrow}$ (thin solid line) The spectra from which the fluxes were extracted are shown in Fig. $1 d$.

when we use the value of $\alpha$ predicted in equation (62). To compare the above predictions for the fluxes (eqs. [65] and [66]) with the fluxes seen in the numerical simulation, we plot the latter in Figure 3. We calculated $\epsilon^{\uparrow}$ with equation (51), using the steady-state spectra shown in Figure $1 d$. The quantity $S^{\downarrow}(p, q)$ that appears in equation $(51)$ is given in equation (49). Since the kinetic code calculates $S^{\downarrow}(p, q)$ in order to evolve the kinetic equations (see eq. [53]), it is trivial to modify the code so that it can be used to evaluate the flux. To calculate $\epsilon^{\downarrow}$, we used the analog of equation (51), with $\uparrow$ and $\downarrow$ switched. We see in Figure 3 that the fluxes are nearly independent of $k$ in the inertial range, with $\epsilon^{\uparrow} \simeq 0.070$ and $\epsilon^{\downarrow} \simeq 0.061$. These values give $\epsilon^{\uparrow} / \epsilon^{\downarrow}-1 \simeq 0.15$, in agreement with equation (66). They also give $\epsilon^{\uparrow} \epsilon^{\downarrow} \simeq 0.0043$, in agreement with equation (65).

From Figure 3, dissipation affects $\epsilon^{\uparrow}$ and $\epsilon^{\downarrow}$ for $k \gtrsim 100$. Thus, dissipation has a large reach. If we add the dissipation term to the flux-conservation form of the kinetic equation (eq. [50]), then we see that in steady state the kinetic equation must satisfy

$$
-\frac{d \epsilon^{\uparrow}}{d \ln k}=\nu k^{4} e^{\uparrow}
$$

The left-hand side of this equation gives the rate of energy increase (per logarithmic band in $k$ ) due to the $k$-space divergence of the flux; in steady state, it must be balanced by the energy loss rate due to dissipation. In Figure 3, we plot $-(d / d \ln k) \epsilon^{\uparrow}$ as a bold dotted line; we evaluated it by taking the numerical derivative of the displayed $\epsilon^{\uparrow}$. The quantity $\nu k^{4} e^{\uparrow}$ is the unlabeled thin dotted line that nearly overlaps $-(d / d \ln k) \epsilon^{\uparrow}$. Thus, equation (67) is satisfied in the simulation. The same is true for the down-waves, which are shown as solid lines in Figure 3. 


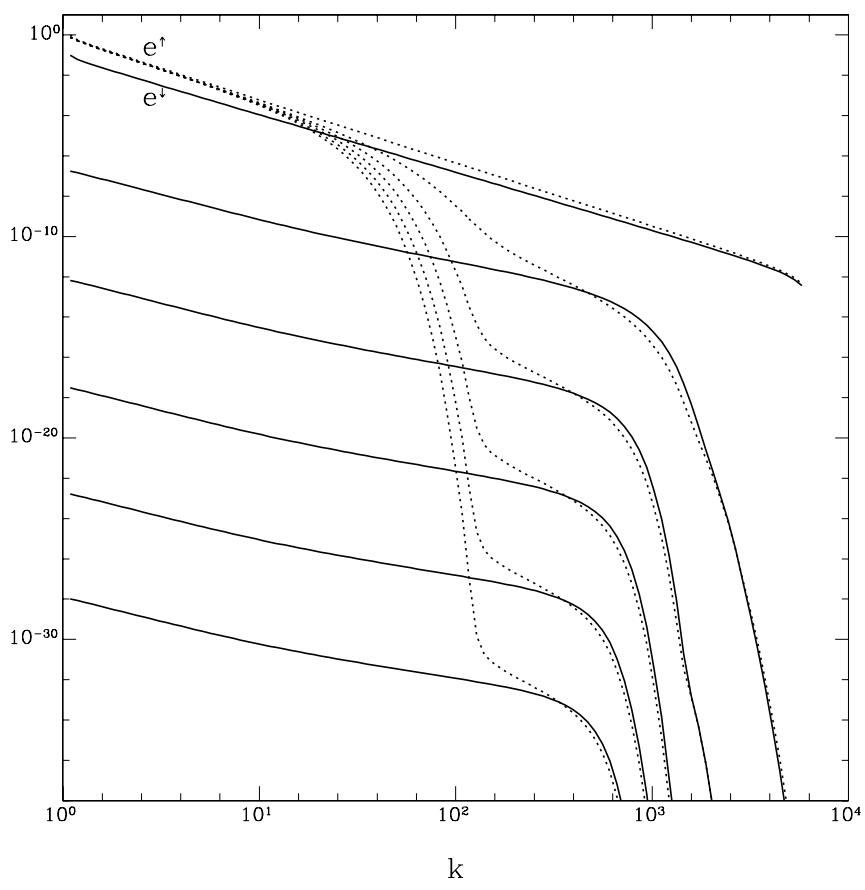

FIG. 4.-Decaying simulation of kinetic equations. Spectra of $e^{\uparrow}$ (dotted lines) and $e^{\downarrow}$ (solid lines) are shown at times $t=0,25,50,75,100$, and 125 (top to bottom).

\subsection{Decaying Turbulence}

For our third simulation, we allow the spectra to decay without injecting any energy. The initial condition is the steady-state spectra from the previous simulation; see Figure 4 . At the outer scale, the cascade time of the downwaves is $\sim 1 / e^{\uparrow}\left(k_{\text {out }}\right) \sim 1$, so $e^{\downarrow}\left(k_{\text {out }}\right)$ decays on this timescale. More precisely, from the values plotted in Figure 4, $e^{\downarrow} \propto \exp (-0.5 t)$ at fixed $k$.

The cascade time of the up-waves is much longer. Initially, $t_{\text {cas }}^{\uparrow} \sim 10$ at the outer scale; as $e^{\downarrow}$ decays, $t_{\text {cas }}^{\uparrow}$ increases exponentially. So $e^{\uparrow}\left(k_{\text {out }}\right)$ does not evolve. At larger $k^{\prime}$ s, $e^{\uparrow}$ is cut off by dissipation; the dissipation wavenumber is $\sim(\nu t)^{-1 / 2} \sim 200 t^{-1 / 2}$.

The spectra appear to evolve in a self-similar manner, with the spectra remaining pinned at their dissipation scale. The end result is that the energy in the down-waves disappears, while the energy in the up-waves is nearly unchanged. Decaying weak turbulence is unstable: an initial imbalance between up- and down-waves is magnified exponentially. A similar instability occurs in strong turbulence, as suggested by Dobrowolny, Mangeney, \& Veltri (1980) in the context of the solar wind, and as seen in numerical simulations of strong turbulence (Maron \& Goldreich 2001; Cho, Lazarian, \& Vishniac 2002).

\section{A MODEL OF THE KINETIC EQUATIONS: COUPLED DIFFUSION EQUATIONS}

It is instructive to model the kinetic equations with two coupled diffusion equations. Investigation of these model equations illustrates that the pinning of spectra is quite general; the only requirement is that the cascade time of one type of wave be inversely related to the energy of the other type. A second reason for considering model equations is that they can be simulated much faster and more easily than the kinetic equations. Finally, by contrasting the kinetic equations with the model equations, we can gain insight into the behavior of the kinetic equations.

\subsection{Derivation of Coupled Diffusion Equations}

Our model equation is

$$
\frac{\partial}{\partial t} k e^{\uparrow}=-\frac{\partial}{\partial k} \tilde{\epsilon}^{\uparrow},
$$

where $k e^{\uparrow}$ is the energy per unit $k$, and $\tilde{\epsilon}^{\uparrow}$ is the flux. This equation is similar in form to equation (50). Recall from $\S 5.1$ that we define the flux as the energy flow rate across a fixed $k \equiv|\boldsymbol{k}|$. It is the "one-dimensional flux," since we average over angles; similarly, $k e^{\uparrow}$ is the "one-dimensional energy density." For $\tilde{\epsilon}^{\uparrow}$, we choose a form that depends on the following quantities evaluated at $k: e^{\downarrow}, e^{\uparrow}$, and $\partial_{k} e^{\uparrow}$. By contrast, the kinetic equations depend on $e^{\uparrow}$ and $e^{\downarrow}$ evaluated at a range of wavenumbers, so unlike the kinetic equations, the diffusion equations are exactly local in $k$. For the cascade time of the energy in the up-waves to have the correct form (eq. [16]), i.e., $t_{\text {cas }}^{\uparrow}=\left(\lambda / w_{\lambda}^{\downarrow}\right)^{2}\left(v_{\mathrm{A}} / \Lambda\right) \sim$ $\left(k^{4} e^{\downarrow}\right)^{-1}\left(v_{\mathrm{A}} / \Lambda\right)$, we choose

$$
\tilde{\epsilon}^{\uparrow}=-\frac{\Lambda}{v_{\mathrm{A}}} e^{\downarrow} k^{6-\beta} \frac{\partial}{\partial k}\left(k^{\beta+1} e^{\uparrow}\right),
$$

where $\beta$ remains to be specified. Of course, the above relation for the flux gives the correct steady-state scaling: $e^{\uparrow} e^{\downarrow} \propto k^{-6}$. The evolution equations for $e^{\downarrow}$ are the same as for $e^{\uparrow}$ (eqs. [68] and [69]), with $\uparrow$ 's and $\downarrow$ 's interchanged.

With $e^{\uparrow} \propto k^{-(3+\alpha)}, e^{\downarrow} \propto k^{-(3-\alpha)}$, we can relate the steadystate fluxes to the energies:

$$
\begin{aligned}
\tilde{\epsilon}^{\uparrow} & =\tilde{f}(\alpha)\left[k^{6} e^{\uparrow}(k) e^{\downarrow}(k)\right] \frac{\Lambda}{v_{\mathrm{A}}}, \\
\tilde{\epsilon}^{\downarrow} & =\tilde{f}(-\alpha)\left[k^{6} e^{\uparrow}(k) e^{\downarrow}(k)\right] \frac{\Lambda}{v_{\mathrm{A}}},
\end{aligned}
$$

where

$$
\tilde{f}(\alpha)=2+\alpha-\beta .
$$

These relations are analogous to equations (29) and (30). To make the analogy closer, we choose $\beta$ so that $f$ has the same dependence on $\alpha$ as does $f$ in the limit that the cascade is nearly balanced (i.e., $|\alpha| \ll 1)$. Since $\tilde{\epsilon}^{\uparrow} / \tilde{\epsilon}^{\downarrow}=\tilde{f}(\alpha) / \tilde{f}(-\alpha)$ $\simeq 1+\alpha /(1-\beta / 2)$ in this limit, we see by comparison with equation (32) that we should choose $\beta=0$.

Collecting results, our model equations are two coupled diffusion equations:

$$
\begin{aligned}
\frac{\partial}{\partial t} e^{\uparrow} & =\frac{\Lambda}{v_{\mathrm{A}}} \frac{1}{k} \frac{\partial}{\partial k}\left(e^{\downarrow} k^{6} \frac{\partial}{\partial k} k e^{\uparrow}\right)-\nu k^{2} e^{\uparrow}, \\
\frac{\partial}{\partial t} e^{\downarrow} & =\frac{\Lambda}{v_{\mathrm{A}}} \frac{1}{k} \frac{\partial}{\partial k}\left(e^{\uparrow} k^{6} \frac{\partial}{\partial k} k e^{\downarrow}\right)-\nu k^{2} e^{\downarrow},
\end{aligned}
$$

after including dissipative terms.

These equations, while much simpler than the kinetic equations, share many of the same features. Since the cascade time of the upgoing waves is inversely proportional to the energy of the downgoing waves (and vice versa), the argument for the pinning of the spectra in weak turbulence (see $\S 3.3$ ) applies here as well. Moreover, in steady state, these diffusion equations suffer from the same degeneracy as does the kinetic equation: the constancy of $\tilde{\epsilon}^{\dagger}$ and $\tilde{\epsilon}^{\downarrow}$ is 
insufficient to determine the scaling of $e^{\uparrow}$ or $e^{\downarrow}$ separately. This degeneracy is partially broken by the dependence of the fluxes on the slopes (eqs. [70] and [71]); we have chosen this dependence so that it is the same as for the kinetic equations in the limit that the cascade is nearly balanced $(|\alpha| \ll 1)$. In the following section, we present numerical simulations of these equations.

\subsection{Numerical Simulations of Coupled Diffusion Equations}

We run two simulations of the coupled diffusion equations. As with the kinetic simulations described in $\S 6$, the first simulation has fixed energy at the outer scale, and the second simulation has fixed flux. And, as before, $2 \Lambda / v_{\mathrm{A}}$ is set to unity, functions of $k$ are evaluated at $k=2^{i / 8}$, $i=1, \ldots, 100$, and the viscosity is $\nu=3 \times 10^{-5}$.

\subsubsection{Fixed Energies at the Outer Scale}

For our first simulation, we fix $e^{\uparrow}\left(k_{\text {out }}\right)=0.63$ and $e^{\downarrow}\left(k_{\text {out }}\right)=0.066 .{ }^{16}$ The evolution is shown in Figure 5. It is very similar to the evolution of the kinetic simulation (Fig. 1). As before, the spectra are pinned quickly. And

\footnotetext{
${ }^{16} \mathrm{We}$ choose these numbers so that the spectra will have the same amplitude here as in the kinetic simulation; see $\S 6.4$.
}

since, by design, the predicted steady state relations for the diffusion equations are the same as those for the kinetic equations (eqs. [61]-[66]), the steady state behavior of the two simulations are nearly identical; compare Figures $5 d$ and $1 d$. There are two differences worthy of note. First, the slopes of $e^{\uparrow}$ and $e \downarrow$ do not have a spike near $k=k_{\text {out }}$. The presence of such a spike in the kinetic simulation is due to the extrapolation of the spectra to $k<k_{\text {out }}$ (see footnote 15). Since the diffusion equations are exactly local in $k$, such an extrapolation is unnecessary, and so the behavior is much smoother near $k=k_{\text {out }}$. Second, in steady state, the diffusion simulations yield spectral slopes that change more gradually as a function of $k$ near the dissipation scale; compare the top panel of Figure $1 d$ with that of Figure $5 d$.

\subsubsection{Fixed Fluxes}

For the second simulation, we inject energy at $k=k_{\text {out }}$ at a fixed rate, equal to that found in steady state in the previous simulation. For the initial condition, we divide the previously found steady-state value of $e^{\uparrow}$ by 10 , and we multiply $e \downarrow$ by 10 . The evolution is shown in Figure 6. It is very similar to that found in the corresponding kinetic simulation (Fig. 2).

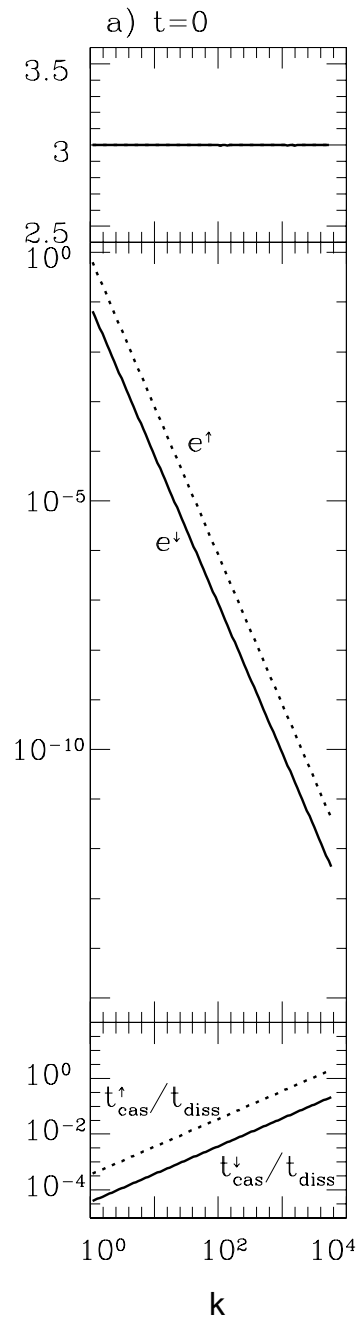

b) $t=0.005$

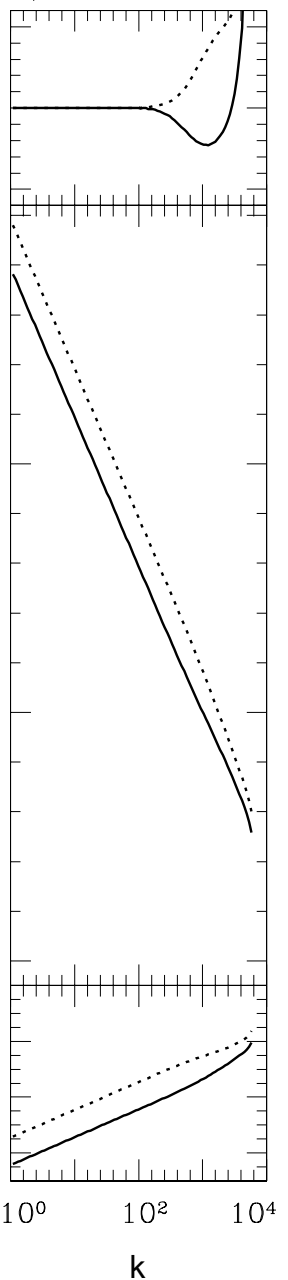

c) $t=0.5$

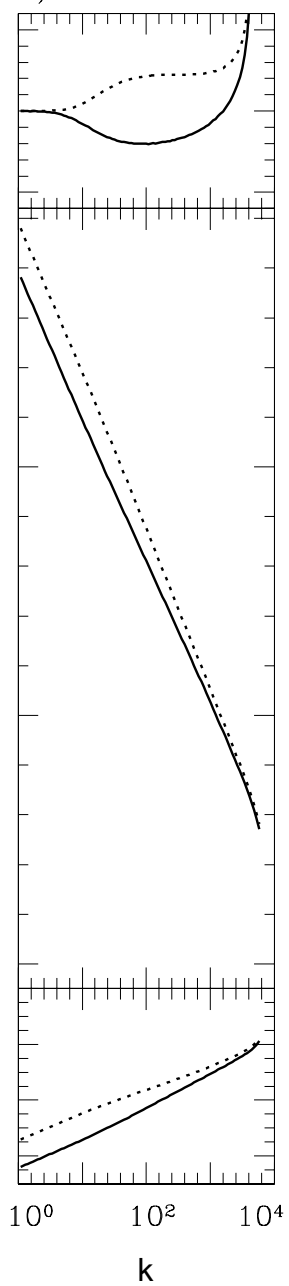

d) $t=50$

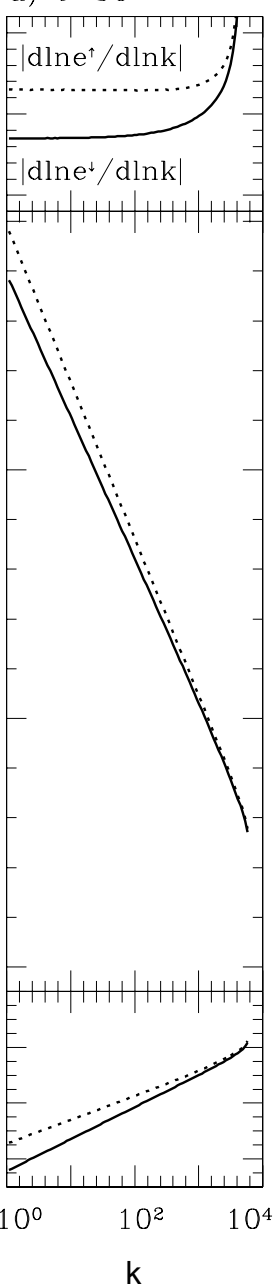

FIG. 5.-Simulation of diffusion equations with fixed energies at the outer scale 
a) $t=0$

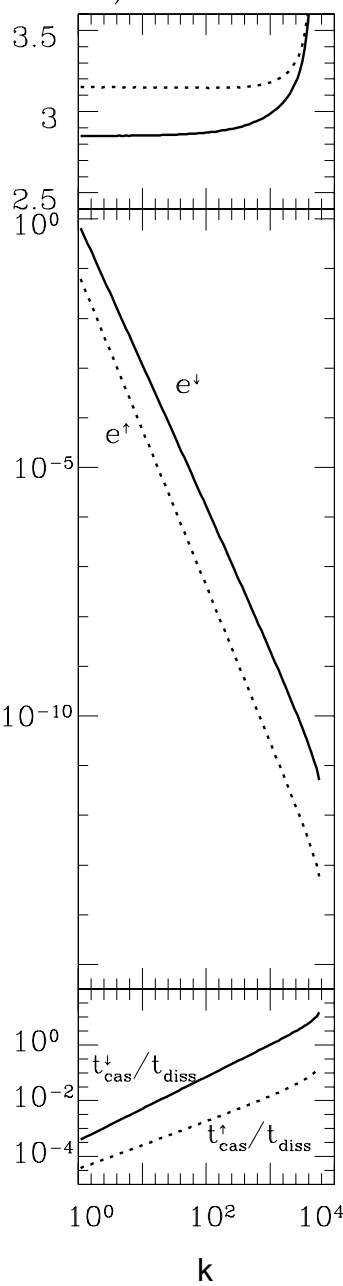

b) $t=0.01$
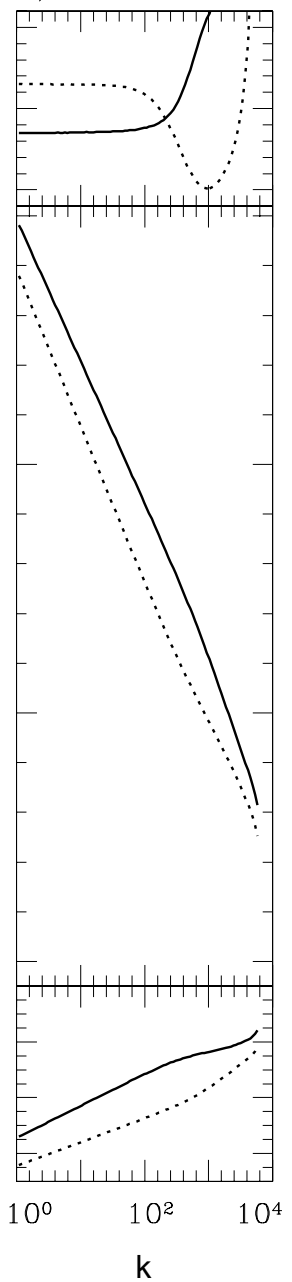

c) $t=0.1$
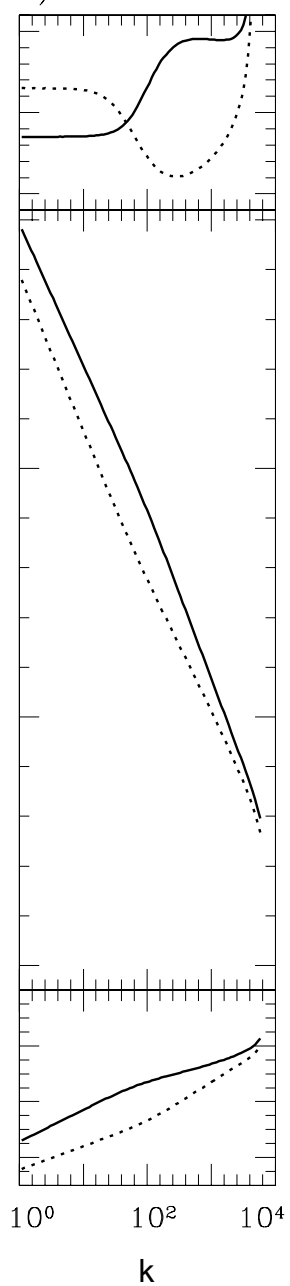

d) $t=500$

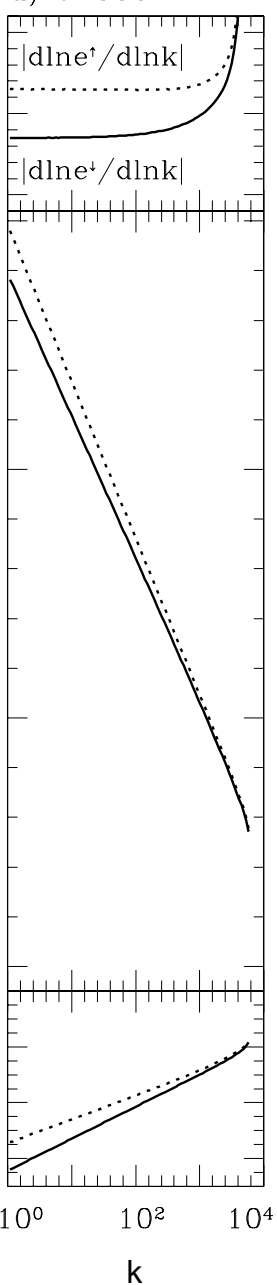

FIG. 6.-Simulation of diffusion equations with fixed fluxes

\section{LARGE MAGNETIC PRANDTL NUMBER}

In incompressible MHD, diffusive dissipation of kinetic and magnetic energies is accounted for by addition of the terms $\nu \nabla^{2} \boldsymbol{v}$ and $\eta \nabla^{2} \boldsymbol{B}$ to the right-hand sides of equations (1) and (2), respectively, where $\nu$ is the viscosity and $\eta$ is the resistivity. The magnetic Prandtl number is defined as

$$
\operatorname{Pr} \equiv \nu / \eta \text {. }
$$

Until now, we have assumed that $\operatorname{Pr}=1$. In this section, we examine weak MHD turbulence when $\operatorname{Pr} \gg 1 .{ }^{17}$

With arbitrary $\nu$ and $\eta$, the equation for $\boldsymbol{w}^{\uparrow}$ (eq. [4]) becomes,

$$
\begin{aligned}
\partial_{t} \boldsymbol{w}^{\uparrow}+v_{\mathrm{A}} \partial_{z} \boldsymbol{w}^{\uparrow}=- & \boldsymbol{w}^{\downarrow} \cdot \boldsymbol{\nabla} \boldsymbol{w}^{\uparrow}-\nabla P+\frac{1}{2}(\nu+\eta) \nabla^{2} \boldsymbol{w}^{\uparrow} \\
& +\frac{1}{2}(\nu-\eta) \nabla^{2} \boldsymbol{w}^{\downarrow} ;
\end{aligned}
$$

the equation for $\boldsymbol{w} \downarrow$ is the same, but with $\uparrow$ and $\downarrow$ interchanged and $v_{\mathrm{A}} \rightarrow-v_{\mathrm{A}}$. To derive the kinetic equation for $e^{\uparrow}$, we first Fourier transform the above equation; the

\footnotetext{
${ }^{17}$ Strong MHD turbulence with $\operatorname{Pr} \gg 1$ occurs in many astrophysical settings.
}

transformed dissipation terms are $-\left(k^{2} / 2\right)(\nu+\eta) \boldsymbol{w}_{k}^{\uparrow}$ $-\left(k^{2} / 2\right)(\nu-\eta) \boldsymbol{w}_{k}^{\downarrow}$. When we take the dot product of the Fourier transformed equation with $\boldsymbol{w}_{k}^{\uparrow}$, and then take the expectation value of the result, we can neglect the dissipation term proportional to $\left\langle\boldsymbol{w}_{k}^{\uparrow} \cdot \boldsymbol{w}_{k}^{\downarrow}\right\rangle$, since it is much smaller than the term proportional to $\left\langle\left(\boldsymbol{w}_{k}^{\uparrow}\right)^{2}\right\rangle$. This is because upgoing waves at fixed $z^{\uparrow} \equiv z-v_{\mathrm{A}} t$ are uncorrelated with the downgoing ones (see $\S$ A.1). As a result, the kinetic equation for high magnetic Prandtl number is the same as that for $\operatorname{Pr}=1$ (eq. [46]), but with $\nu k^{2} e^{\uparrow}$ replaced by $(\nu+\eta) k^{2} e^{\uparrow} / 2 \simeq \nu k^{2} e^{\uparrow} / 2$. This might appear to be a surprising result. In the limit of vanishing $\eta$, the energy spectra are cut off below the viscous scale, i.e., the scale set by $\nu$ (eq. [27], within a factor of 2). One might have expected that the viscous scale should only affect the kinetic energy spectrum. Yet the magnetic energy spectrum is also cut off below the viscous scale. This can be understood as follows: upgoing waves each have equal magnetic and kinetic energies, and similarly for downgoing waves. As an up-wave with length scale slightly larger than the viscous scale is gradually cascaded to subviscous scales, its kinetic energy is dissipated by viscosity. Since the cascade time in weak turbulence is much longer than the waveperiod, as the up-wave's kinetic energy is dissipated, its magnetic energy is converted into kinetic 
energy so that its magnetic and kinetic energies can remain nearly equal. As a result, both kinetic and magnetic energies are dissipated at the viscous scale.

\section{HYPERVISCOSITY AND THE BOTTLENECK EFFECT}

In many different types of turbulent cascades, the energy spectrum exhibits a hump on scales slightly larger than the dissipation scale (e.g., Falkovich 1994). The hump is particularly pronounced in numerical simulations that use "hyperviscosity," a trick whereby the diffusive term $\nu k^{2} v$ is replaced by $\nu_{n} k^{n} \boldsymbol{v}$, where $n$ is typically 4 or 8 , and $\nu_{n}$ is the hyperviscosity; the resistivity is modified in a similar manner (e.g., Borue \& Orszag 1995; Biskamp 2000). With this trick, a smaller part of the spectrum is affected by dissipation, so a longer inertial range can be simulated with a fixed resolution. Although this trick does work, there is a problem: the spectrum on scales slightly larger than the dissipation scales is made flatter. The energy, in effect, is backed up. This is the bottleneck effect. It can be particularly problematic in simulations of strong MHD turbulence, where the energy backup can affect length scales considerably larger than the dissipative scales (Biskamp \& Müller 2000).

This motivates us to investigate the bottleneck effect in weak turbulence. If the bottleneck effect appears, its interpretation will be simpler than in strong turbulence. We perform two simulations of the kinetic equation, one with hyperviscosity of the form $\nu_{4} k^{4}$, and the other with $\nu_{8} k^{8}$. In each simulation, we fix the energies at the outer scale, $e^{\uparrow}\left(k_{\text {out }}\right)=1$, $e^{\downarrow}\left(k_{\text {out }}\right)=0.1$, and allow the spectra to reach steady state. The steady-state spectra of $k^{6} e^{\uparrow} e^{\downarrow}$ are shown in Figure 7, offset for clarity. Also shown is the simulation with ordinary viscosity described in $\S 6.2$. From this figure it is apparent that weak turbulence suffers from the bottleneck effect; the effect becomes larger with increasing hyperviscous exponent.

The bottleneck effect can be understood as follows. Consider an up-wave with a length scale slightly larger than the dissipation scale. It is cascaded by down-waves that have slightly different length scales than its own. Hyperviscosity gives a sharper dissipative cutoff to the down-wave spectrum than ordinary viscosity. Therefore, a hyperviscous simulation has fewer down-waves in the vicinity of the dissipation scale, and the cascade time of the up-wave is longer. For the up-wave energy flux to be independent of length scale, a longer cascade time implies a larger energy. As a result, the spectrum is flatter on scales slightly larger than dissipative ones. Falkovich (1994) offers a similar explanation for the bottleneck effect in hydrodynamic hydroturbulence.

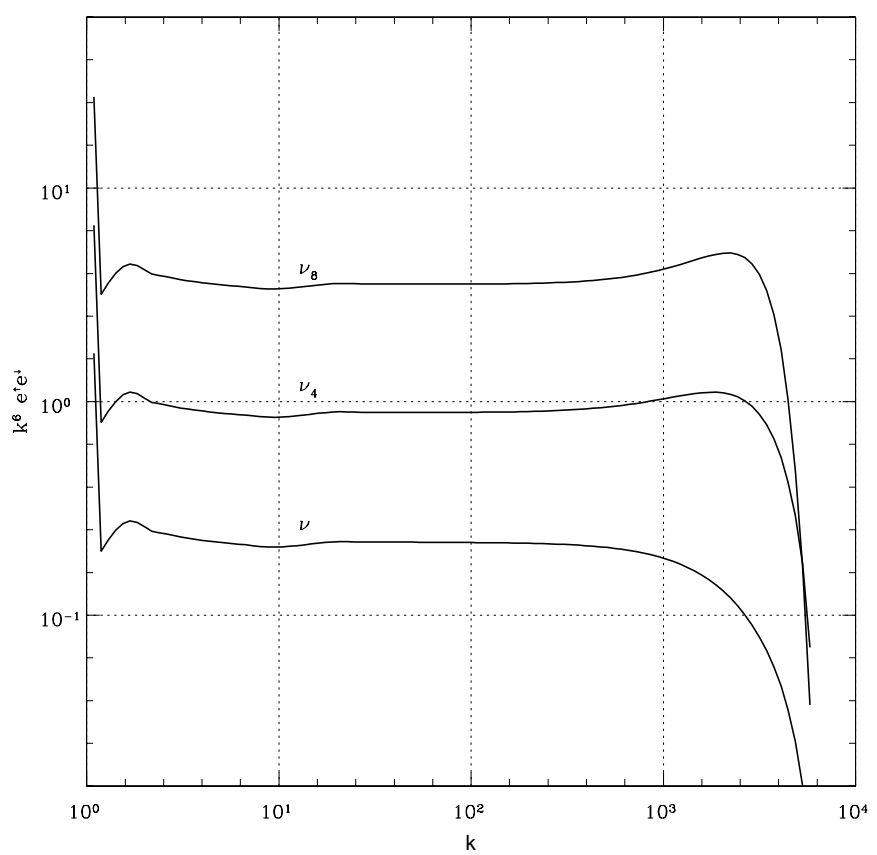

FIG. 7.-Bottleneck effect. Three spectra are shown, offset for clarity: top spectrum has viscous and resistive terms $\nu_{8} k^{8}$, the middle has $\nu_{4} k^{4}$, and the bottom has $\nu k^{2}$.

\section{DISCUSSION}

In this paper we describe imbalanced weak turbulence and solve the steady-state cascade. In a future paper, we will extend the result to the strong cascade. One of our ultimate goals is to develop a theory of imbalanced strong turbulence to apply to the solar wind, where imbalance is observed.

Although strong turbulence is more generally applicable than weak, the latter is a simple and illuminating model. There are a number of issues in strong turbulence that are not understood. Weak turbulence can be used as the first step in explaining them. For example, in this paper we examined the effect of a large magnetic Prandtl number on weak MHD turbulence. We also found that the bottleneck effect appears in weak turbulence, where its interpretation is straightforward. There are a number of other issues that we intend to investigate in weak turbulence as a prelude to understanding them in strong turbulence; for example, reconnection and the turbulent dynamo.

Research reported in this paper was supported by NSF grant AST 00-98301.

\section{APPENDIX}

\section{KINETIC EQUATION IN WEAK TURBULENCE}

\section{A1. PRELIMINARIES}

Kinetic equations describing weak MHD turbulence are derived by Galtier et al. (2000, 2002). In this appendix, we present a more physical derivation. We compare the two derivations in $\S$ A.5.

We consider the evolution of $\boldsymbol{w}^{\uparrow}$ in a plane that is transverse to $\hat{\boldsymbol{z}}$ and moving with velocity $v_{\mathrm{A}} \hat{\boldsymbol{z}}$, i.e., with fixed $z^{\uparrow} \equiv z-v_{\mathrm{A}} t$. Recall that $\hat{z}$ is in the direction of the mean magnetic field. Changing variables from $z$ to $z^{\uparrow}$ in equation (8) gives

$$
\left.\frac{\partial}{\partial t}\right|_{x, y, z^{\downarrow}} \boldsymbol{w}^{\uparrow}=-\boldsymbol{w}^{\downarrow} \cdot \nabla_{\perp} \boldsymbol{w}^{\uparrow}+\nabla_{\perp} \nabla_{\perp}^{-2}\left(\nabla_{\perp} \boldsymbol{w}^{\downarrow}: \boldsymbol{\nabla}_{\perp} \boldsymbol{w}^{\uparrow}\right) .
$$


In weak turbulence, the parallel length over which disturbances are correlated, $\Lambda$, is small; in particular, in the time that an upwave crosses a downgoing wavepacket, $\Delta t \sim \Lambda / v_{\mathrm{A}}$, its distortion is less than unity: $\Delta w_{\lambda}^{\uparrow} / w_{\lambda}^{\uparrow} \sim\left(w_{\lambda}^{\downarrow} / v_{\mathrm{A}}\right) \Lambda / \lambda \ll 1$ (eq. [15]). ${ }^{18}$ Therefore, $\boldsymbol{w}^{\uparrow}$ undergoes small uncorrelated changes each $\Delta t \sim \Lambda / v_{\mathrm{A}}$, and its evolution is analogous to a random walk, with step size $\Delta w_{\lambda}^{\uparrow} \sim w_{\lambda}^{\uparrow}\left(w_{\lambda}^{\downarrow} / v_{\mathrm{A}}\right) \Lambda / \lambda \ll w_{\lambda}^{\uparrow}$. Our goal in this appendix is to quantify the random walk; the resulting equation is the kinetic equation. The kinetic equation is only valid when the turbulence is weak: its derivation hinges on the assumption that the time for up-waves to cascade is longer than the correlation time of down-waves at fixed $z^{\uparrow}$, i.e., that $\left(w_{\lambda}^{\downarrow} / v_{\mathrm{A}}\right) \Lambda / \lambda \ll 1$.

Equation (A1) is linear in $\boldsymbol{w}^{\uparrow}$. Nonlinearity arises because $\boldsymbol{w}^{\uparrow}$ modifies $\boldsymbol{w} \downarrow$ (through eq. [10]); this modification back-reacts on $\boldsymbol{w}^{\uparrow}$ through equation (A1). Nonetheless, this back-reaction can be neglected when deriving the kinetic equation. The reason is as follows. Every $\Delta t \sim \Lambda / v_{\mathrm{A}}$, the back-reaction changes $w_{\lambda}^{\uparrow}$ by $\sim w_{\lambda}^{\uparrow}\left[\left(w_{\lambda}^{\downarrow} / v_{\mathrm{A}}\right) \Lambda / \lambda\right]\left[\left(w_{\lambda}^{\uparrow} / v_{\mathrm{A}}\right) \Lambda / \lambda\right]$, which is smaller than $\Delta w_{\lambda}^{\uparrow}$ by the small factor $\left(w_{\lambda}^{\uparrow} / v_{\mathrm{A}}\right) \Lambda / \lambda \ll 1$. Furthermore, since this back-reaction is uncorrelated on timescales larger than $\Lambda / v_{\mathrm{A}}$, it only effects a small change in the step size of the random walk.

Therefore, in deriving the kinetic equation, we interpret equation (A1) as a linear equation for $\boldsymbol{w}^{\uparrow}$ (at fixed $z^{\uparrow}$ ); $\boldsymbol{w}^{\downarrow}$ at fixed $z^{\uparrow}$ is viewed as a function with known statistical properties. To simplify our derivation, we first solve a model problem: the linear random oscillator. The extension to weak turbulence will then be straightforward.

\section{A2. A TOY PROBLEM: THE LINEAR RANDOM OSCILLATOR}

We consider the evolution of a simple random oscillator $\psi$ :

$$
\frac{d}{d t} \psi(t)=i A(t) \psi(t),
$$

where $\psi$ is a complex scalar and $A$ is a real random variable; the factor $i$ ensures that the energy $|\psi(t)|^{2}$ is conserved. ${ }^{19}$ Our goal is to calculate the evolution of $\langle\psi(t)\rangle$, where angle brackets denote an average over an ensemble of $A$ 's, not over time. We assume that the values of $A$ at two different times are statistically independent of each other whenever the two times are separated by more than the correlation time, $\tau_{\text {corr }}$; we make this more precise in footnote 21 . For simplicity, we take $A$ to have zero mean, $\langle A(t)\rangle=0$; the extension to $A$ with nonzero mean is trivial. We assume that the statistical properties of $A$ such as $\tau_{\text {corr }}$ and $A_{\text {rms }} \equiv\left\langle A^{2}\right\rangle^{1 / 2}$ - vary only on timescales much larger than $\tau_{\text {corr }}$. Note that $\psi$ corresponds to $w_{\lambda}^{\uparrow}$ in equation (A1); $A$ corresponds to $w_{\lambda}^{\downarrow}$, or, more specifically, to $w_{\lambda}^{\downarrow} / \lambda$; and $\tau_{\text {corr }}$ corresponds to $\Lambda / v_{\mathrm{A}}$.

There are two limiting regimes for the random oscillator, depending on whether $A_{\mathrm{rms}} \tau_{\mathrm{corr}}$ is less than or greater than unity. In the following, we take $A_{\mathrm{rms}} \tau_{\text {corr }} \ll 1$; this regime corresponds to weak turbulence. The change in $\psi$ within the time $\tau_{\text {corr }}$ is of order $A_{\text {rms }} \tau_{\text {corr }} \psi$, which is much smaller than $\psi$. Thus, the correlation time of $A$ is smaller than the "cascade time" of $\psi$. Since $A$ is uncorrelated on timescales larger than $\tau_{\text {corr }}, \psi$ undergoes small uncorrelated changes each $\tau_{\text {corr }}$, and thus its long-time evolution is a random walk. Intuitively, we expect that the time evolution of the statistical properties of $\psi$-in particular, $\langle\psi\rangle$ - can be represented by a differential equation. Our goal in this section is to derive the differential equation, and to understand the approximations that are made in deriving it.

The solution of equation (A2) is simply

$$
\psi(t)=\psi(0) \exp \left(i A^{t}\right)
$$

where $A^{t} \equiv \int_{0}^{t} A\left(t^{\prime}\right) d t^{\prime}$. We expand as

$$
\psi(t)=\psi(0)\left[1+i A^{t}-\left(A A^{t}\right)^{t}+\ldots\right],
$$

where $\left(A A^{t}\right)^{t} \equiv \int_{0}^{t} A\left(t^{\prime}\right) \int_{0}^{t^{\prime}} A\left(t^{\prime \prime}\right) d t^{\prime \prime} d t^{\prime}$; note that $(1 / 2)\left(A^{t}\right)^{2}=\left(A A^{t}\right)^{t}$ is an identity for any $A(t)$. Equation (A4) can be thought of as an expansion in powers of $A$; it is only valid for short times after $t=0$.

We evaluate $\langle\psi(t)\rangle$ as follows. Since $A(t)$ is unaffected by $\psi(0), A(t)$ and $\psi(0)$ are uncorrelated ${ }^{20}$ So

$$
\langle\psi(t)\rangle=\langle\psi(0)\rangle\left(1+i\left\langle A^{t}\right\rangle-\left\langle\left(A A^{t}\right)^{t}\right\rangle+\langle\ldots\rangle\right) \text {. }
$$

Since $\langle A\rangle=0$, we have $\left\langle A^{t}\right\rangle=0$. Next, we consider $\left\langle\left(A A^{t}\right)^{t}\right\rangle$. The values of $A$ at two different times "statistically overlap" only when the two times are separated by less than the correlation time; i.e., $\left\langle A\left(t^{\prime}\right) A\left(t^{\prime}+\Delta t\right)\right\rangle$ is nonzero only if $\Delta t<\tau_{\text {corr }}$. Thus, $\left\langle A A^{t}\right\rangle \simeq A_{\mathrm{rms}}^{2} \tau_{\text {corr }}$ when $t \gtrsim \tau_{\text {corr }}$, and ${ }^{21}$

$$
\left\langle\left(A A^{t}\right)^{t}\right\rangle \simeq A_{\mathrm{rms}}^{2} \tau_{\mathrm{corr}} t
$$

Therefore,

$$
\langle\psi(t)\rangle \simeq\langle\psi(0)\rangle\left(1-A_{\mathrm{rms}}^{2} \tau_{\text {corr }} t\right), \quad \tau_{\text {corr }} \lesssim t \ll \tau_{\text {corr }} /\left(A_{\text {rms }} \tau_{\text {corr }}\right)^{2}
$$

\footnotetext{
${ }^{18}$ We neglect the factor of 2 associated with the fact that the relative velocity of up and down waves is $2 v_{\mathrm{A}}$.

${ }^{19}$ For a textbook discussion of the linear random oscillator, see van Kampen (1992).

${ }^{20} \mathrm{In}$ fact, $A(t>0)$ and $\psi(0)$ are slightly correlated: $\psi(0)$ is affected by $A\left(t_{1}<0\right)$, which is in turn correlated with $A\left(t_{2}>0\right)$ as long as $t_{2}-t_{1} \lesssim \tau_{\text {corr }}$. Nonetheless, in the limit $A_{\text {rms }} \tau_{\text {corr }} \ll 1$ that we are considering, $\psi$ only changes by a small amount in the time $\tau_{\text {corr }}$. So the correlation between $A\left(0<t \lesssim \tau_{\text {corr }}\right)$ and $\psi(0)$ is unimportant for the evolution of $\psi(t)$.

${ }^{21}$ For the following equation to be approximately valid, $A$ must decorrelate sufficiently rapidly that $\left\langle A\left(t^{\prime}\right) A\left(t^{\prime}+\Delta t\right)\right\rangle$ goes to zero faster than $1 / \Delta t$ for large $\Delta t>\tau_{\text {corr }}$; otherwise, $\left\langle\left(A A^{t}\right)^{t}\right\rangle$ rises faster than the first power of $t$, and our derivation is invalid.
} 
Equivalently,

$$
\frac{d}{d t}\langle\psi(t)\rangle=-A_{\mathrm{rms}}^{2} \tau_{\mathrm{corr}}\langle\psi(t)\rangle, \quad \text { assuming } A_{\mathrm{rms}} \tau_{\text {corr }} \ll 1 .
$$

This equation is the main result of this section. Van Kampen (1992) gives a more mathematically rigorous derivation of it than we do. Equation (A8) can be understood as follows: since $\psi$ changes by $\Delta \psi \sim A_{\text {rms }} \tau_{\text {corr }} \psi$ in the time $\tau_{\text {corr }}$, then after $N$ steps, each $\tau_{\text {corr }}$ long, the change in $\psi$ is $\sqrt{N} \Delta \psi$. Thus, for order unity changes in $\psi,(\psi / \Delta \psi)^{2}$ steps are required. The resulting time, the "cascade time," is

$$
\tau_{\text {cas }}=(\psi / \Delta \psi)^{2} \tau_{\text {corr }} \sim 1 /\left(A_{\text {rms }}^{2} \tau_{\text {corr }}\right),
$$

as in equation (A8), and $\tau_{\text {corr }} / \tau_{\text {cas }} \sim\left(A_{\text {rms }} \tau_{\text {corr }}\right)^{2} \ll 1$. Although $\langle\psi(t)\rangle$ decays to zero on the timescale $\tau_{\text {cas }}$, the energy $|\psi|^{2}$ remains constant; the "cascade" of $\langle\psi\rangle$ is analogous to phase mixing.

We can now proceed to derive the kinetic equation in weak turbulence. Before doing so, we consider the linear random oscillator in more detail. If the reader is satisfied with the above derivation of equation (A8), the following subsection may be skipped.

\section{A2.1. The Validity of Perturbation Theory and Goldreich \& Sridhar (1997)}

Goldreich \& Sridhar (1997) incorrectly claim that perturbation theory fails in weak turbulence, i.e., in their " intermediate" turbulence. ${ }^{22}$ We explain their claim and its resolution in the context of the linear random oscillator. In the process, we clarify the validity of the perturbation expansion.

We consider the terms neglected in equation (A7). For example, the fourth-order term is $\left\langle\psi^{(4)}\right\rangle /\langle\psi(0)\rangle=\left\langle\left\{A\left[A\left(A A^{t}\right)^{t}\right]^{t}\right\}^{t}\right\rangle$. In this quadruple time integral, $A$ is evaluated at four different times. Whenever two of these times are separated by less than $\tau_{\text {corr }}$, the values of $A$ at these two times "statistically overlap," and hence can give a nonzero contribution to the total integral. If two of the times are separated by less than $\tau_{\text {corr }}$, and the other two times are also separated by less than $\tau_{\text {corr }}$, then the integrand does not vanish, even if the first two times are separated from the second two times by more than $\tau_{\text {corr }}$. So the quadruple time integral yields approximately $\langle\psi(4)\rangle /\langle\psi(0)\rangle \sim\left(A_{\text {rms }}^{2} \tau_{\text {corr }} t\right)^{2}=\left(t / \tau_{\text {cas }}\right)^{2}$.

Since $\left\langle\psi^{(2)}\right\rangle /\langle\psi(0)\rangle=-A_{\mathrm{rms}}^{2} \tau_{\text {corr }} t=-t / \tau_{\text {cas }}$, the contribution of the fourth-order term is as large as the second-order term after the time $t=\tau_{\text {cas }}$. Similarly, if we consider the contribution to $\left\langle\psi^{(2 n)}\right\rangle /\langle\psi(0)\rangle$ from correlating pairs of $A$, the result is $\sim\left(t / \tau_{\text {cas }}\right)^{n}$; so all terms are of comparable value when $t=\tau_{\text {cas }}$, and it seems that the lowest order term is inadequate. This, in effect, is the claim that Goldreich \& Sridhar (1997) make in the context of weak turbulence.

It is incorrect; although equation (A7) for $\langle\psi(t)\rangle$ is only valid for $t \ll \tau_{\text {cas }}$, equation (A8) for $(d / d t)\langle\psi(t)\rangle$ is approximately valid for all times, with corrections of order powers of $\tau_{\text {corr }} / \tau_{\text {cas }} \ll 1$. Since $\psi(t)$ undergoes small uncorrelated changes every time step of length $\tau_{\text {corr }}$, we expect on physical grounds that the evolution of its statistical properties should be governed by a differential equation that is invariant under time translations. ${ }^{23}$ Equation (A8) is the only such equation whose small-time behavior is given by equation (A7). Its right-hand side can be interpreted as the lowest term in a perturbative expansion in $\tau_{\text {corr }} / \tau_{\text {cas }}$. All of the contributions to $\langle\psi(t)\rangle$ that are of order $\left(t / \tau_{\text {cas }}\right)^{n}$ must be derivable from equation (A8). For example, if $A_{\mathrm{rms}}^{2} \tau_{\text {corr }} \equiv 1 / \tau_{\text {cas }}$ is constant, then equation (A8) has the solution $\langle\psi(t)\rangle /\langle\psi(0)\rangle=\exp \left(-t / \tau_{\text {cas }}\right)=$ $1-t / \tau_{\text {cas }}+(1 / 2)\left(t / \tau_{\text {cas }}\right)^{2}+\ldots$. Terms of order $\left(t / \tau_{\text {cas }}\right)^{n}$ in $\langle\psi\rangle$ are generated from the lowest order term.

We can solve the one-dimensional oscillator exactly when $A(t)$ is Gaussian, and thereby illustrate the validity of equation (A8). If $A(t)$ is a zero-mean Gaussian random variable, then so is $A^{t}$, and $\left\langle\exp \left(i A^{t}\right)\right\rangle=\exp \left[-\left\langle\left(A^{t}\right)^{2}\right\rangle / 2\right]{ }^{24}$ It follows from equation (A3) that

$$
\langle\psi(t)\rangle=\langle\psi(0)\rangle \exp \left[-\left\langle\left(A A^{t}\right)^{t}\right\rangle\right],
$$

assuming that $\langle\psi(0)\rangle$ and $A(t)$ are uncorrelated. Equivalently,

$$
\frac{d}{d t}\langle\psi\rangle=-\left\langle A A^{t}\right\rangle\langle\psi\rangle
$$

which agrees with equation (A8) when $\left\langle A A^{t}\right\rangle=A_{\mathrm{rms}}^{2} \tau_{\text {corr }}$.

Although we are mainly concerned with the limit $A_{\mathrm{rms}} \tau_{\text {corr }} \ll 1$, we conclude this subsection by briefly discussing the opposite limit, $A_{\text {rms }} \tau_{\text {corr }} \gg 1$. This limit sheds some light on strong turbulence, which corresponds to the case $A_{\text {rms }} \tau_{\text {corr }} \sim 1$. When $A_{\text {rms }} \tau_{\text {corr }} \gg 1$, perturbation theory is inapplicable because $\psi$ does not undergo small uncorrelated changes every $\tau_{\text {corr }}$. Rather, its cascade time is $\sim 1 / A_{\mathrm{rms}}$, which is much shorter than the correlation time of $A$. Therefore, $A$ is nearly constant in the time that $\langle\psi\rangle$ cascades. The inapplicability of perturbation theory makes strong turbulence difficult, if not impossible, to solve. Nonetheless, we can solve the one-dimensional oscillator in the "strong" limit. Equations (A10) and (A11) are still valid, but $\left\langle A A^{t}\right\rangle \sim A_{\mathrm{rms}}^{2} t$, so $\langle\psi(t)\rangle \sim\langle\psi(0)\rangle \exp \left(-A_{\mathrm{rms}}^{2} t^{2} 2\right)$, and equation (A11) is not invariant under time translations. The time $t=0$

\footnotetext{
${ }^{22}$ A note on terminology: the turbulence that we and that Galtier et al. (2000) call "weak," Goldreich \& Sridhar (1997) call " intermediate." They call it "intermediate" precisely because of their claim that perturbation theory is invalid.

${ }^{23}$ It should be invariant on the timescale $\tau_{\text {corr }} ; A_{\text {rms }}$ and $\tau_{\text {corr }}$ are effectively constants on this timescale.

24 Proof: a zero-mean Gaussian $x$ has probability distribution $P(x)=\left(2 \pi x_{\mathrm{rms}}^{2}\right)^{-1 / 2} \exp \left(-x^{2} / 2 x_{\mathrm{rms}}^{2}\right) ; \operatorname{so}\langle\exp (i x)\rangle=\int_{-\infty}^{\infty} P(x) \exp (i x) d x=\exp \left(-x_{\mathrm{rms}}^{2} / 2\right)$.
} 
is special because of our assumption that $\psi(0)$ and $A(t)$ are uncorrelated. This assumption, while innocuous for the "weak" oscillator, is crucial for the "strong" one.

\section{A3. DERIVATION OF THE KINETIC EQUATION}

We derive the kinetic equation in Fourier space. We Fourier transform equation (A1) in $x$ and $y$ (but not $z$ ), denoting the transform of $\boldsymbol{w}^{\uparrow}$ by $\boldsymbol{w}_{\boldsymbol{k}}^{\uparrow}$ :

$$
\boldsymbol{w}_{\boldsymbol{k}}^{\uparrow} \equiv \int d^{2} \boldsymbol{x}_{\perp} \boldsymbol{w}^{\uparrow} \exp \left(-i \boldsymbol{k} \cdot \boldsymbol{x}_{\perp}\right)
$$

where $\boldsymbol{k}$ is purely transverse $\left(k_{z} \equiv 0\right)$; similarly, $\boldsymbol{w}_{\boldsymbol{k}}^{\downarrow}$ is the Fourier transform of $\boldsymbol{w} \downarrow$.

Since $\boldsymbol{w}_{\boldsymbol{k}}$ is perpendicular to both $\boldsymbol{k}$ and $\hat{\boldsymbol{z}}$, it only represents a single degree of freedom. Hence, it is convenient to define a scalar potential $\psi_{\boldsymbol{k}}^{\uparrow}$ by $\boldsymbol{w}_{\boldsymbol{k}}^{\uparrow}=i(\hat{\boldsymbol{k}} \times \hat{\boldsymbol{z}}) \psi_{\boldsymbol{k}}^{\uparrow}$, with $\hat{\boldsymbol{k}} \equiv \boldsymbol{k} / k$. Similarly, $\boldsymbol{w}_{\boldsymbol{k}}^{\downarrow}=i(\hat{\boldsymbol{k}} \times \hat{\boldsymbol{z}}) \psi_{\boldsymbol{k}}^{\downarrow}$. The Fourier transform of equation (A1) is then

$$
\left.\frac{\partial}{\partial t}\right|_{\boldsymbol{k}, \tau^{\ddagger}} \psi_{\boldsymbol{k}}^{\uparrow}(t)=\int d^{2} \boldsymbol{p} A_{\boldsymbol{k}, \boldsymbol{p}}(t) \psi_{\boldsymbol{p}}^{\uparrow}(t)
$$

where

$$
\begin{aligned}
A_{\boldsymbol{k}, \boldsymbol{p}}(t) & \equiv a_{\boldsymbol{k}, \boldsymbol{p}} \psi_{\boldsymbol{k}-\boldsymbol{p}}^{\downarrow}(t) \\
& \equiv \frac{1}{(2 \pi)^{2}} \hat{\boldsymbol{z}} \cdot(\boldsymbol{k} \times \boldsymbol{p}) \hat{\boldsymbol{k}} \cdot \hat{\boldsymbol{p}} \frac{\psi_{\boldsymbol{k}-\boldsymbol{p}}^{\downarrow}(t)}{|\boldsymbol{k}-\boldsymbol{p}|} .
\end{aligned}
$$

We suppress the functional dependences of $\psi^{\uparrow}$ and $\psi \downarrow$ on $z^{\uparrow}$ because this equation is evaluated at fixed $z^{\uparrow}$; in the following, we replace the partial time derivative by a total derivative, with the understanding that $z^{\uparrow}$ is fixed. ${ }^{25}$

We use angle brackets to denote an ensemble average, in a plane with fixed $z^{\uparrow}$. We define the spectral energy densities $e^{\uparrow}$ and $e^{\downarrow}$ as

$$
\begin{aligned}
& \left\langle\boldsymbol{w}_{\boldsymbol{k}}^{\uparrow}(t) \cdot \boldsymbol{w}_{\boldsymbol{k}^{\prime}}^{\uparrow}(t)\right\rangle=\left\langle\psi_{\boldsymbol{k}}^{\uparrow}(t) \psi_{\boldsymbol{k}^{\prime}}^{\uparrow}(t)\right\rangle=e^{\uparrow}(\boldsymbol{k}, t) \delta\left(\boldsymbol{k}+\boldsymbol{k}^{\prime}\right) \\
& \left\langle\boldsymbol{w}_{\boldsymbol{k}}^{\downarrow}(t) \cdot \boldsymbol{w}_{\boldsymbol{k}^{\prime}}^{\downarrow}(t)\right\rangle=\left\langle\psi_{\boldsymbol{k}}^{\downarrow}(t) \psi_{\boldsymbol{k}^{\prime}}^{\downarrow}(t)\right\rangle=e^{\downarrow}(\boldsymbol{k}, t) \delta\left(\boldsymbol{k}+\boldsymbol{k}^{\prime}\right),
\end{aligned}
$$

where $e^{\uparrow}$ and $e^{\downarrow}$ are real; $\delta(\boldsymbol{k})$ is a two-dimensional Dirac delta function that follows from homogeneity, i.e., from the assumption that $\left\langle\boldsymbol{w}^{\uparrow}\left(\boldsymbol{x}_{\perp}\right) \cdot \boldsymbol{w}^{\uparrow}\left(\boldsymbol{x}_{\perp}+\Delta \boldsymbol{x}_{\perp}\right)\right\rangle$ is independent of $\boldsymbol{x}_{\perp}$.

We derive the evolution equation for the bilinear quantity $\left\langle\psi_{\boldsymbol{k}}^{\uparrow} \psi_{\boldsymbol{k}^{\prime}}^{\uparrow}\right\rangle$. From equation (A13),

$$
\frac{d}{d t}\left(\psi_{\boldsymbol{k}}^{\uparrow} \psi_{\boldsymbol{k}^{\prime}}^{\uparrow}\right)=\int d^{2} \boldsymbol{p} A_{\boldsymbol{k}, \boldsymbol{p}}\left(\psi_{\boldsymbol{k}^{\prime}}^{\uparrow} \psi_{\boldsymbol{p}}^{\uparrow}\right)+\left(\boldsymbol{k} \leftrightarrow \boldsymbol{k}^{\prime}\right)
$$

where $\left(\boldsymbol{k} \leftrightarrow \boldsymbol{k}^{\prime}\right)$ represents a second term that is the same as the first, but with $\boldsymbol{k}$ and $\boldsymbol{k}^{\prime}$ interchanged.

From $\S$ A.1, $\psi \downarrow$ (and hence $A_{k, p}$ ) can be viewed as evolving independently of $\psi^{\uparrow}$, since the back-reaction is negligible at fixed $z^{\uparrow}$. Therefore, equation (A18) is nearly identical to that of the simple random oscillator (eq. [A2]); $\left(\psi_{\boldsymbol{k}}^{\uparrow} \psi_{\boldsymbol{k}^{\prime}}^{\uparrow}\right)$ and $A_{\boldsymbol{k}, \boldsymbol{p}}$ in the above equation correspond to $\psi$ and $A$, respectively, in equation (A2). Since $\psi \downarrow$ is a random function with zero mean and correlation time $\tau_{\text {corr }}=\Lambda / v_{\mathrm{A}}$, so is $A_{\boldsymbol{k}, \boldsymbol{p}}$. We solve equation (A18) it in the same manner as we solved the random oscillator, using perturbation theory. Expanding in $A_{\boldsymbol{k}, \boldsymbol{p}}$, we find to zeroth order:

$$
\left(\psi_{\boldsymbol{k}}^{\uparrow} \psi_{\boldsymbol{k}^{\prime}}^{\uparrow}\right)^{(0)}=\mathrm{constant}
$$

To first order,

$$
\left(\psi_{\boldsymbol{k}}^{\uparrow} \psi_{\boldsymbol{k}^{\prime}}^{\uparrow}\right)^{(1)}=\int d^{2} \boldsymbol{p} A_{\boldsymbol{k}, \boldsymbol{p}}^{t}\left(\psi_{\boldsymbol{k}^{\prime}}^{\uparrow} \psi_{\boldsymbol{p}}^{\uparrow}\right)^{(0)}+\left(\boldsymbol{k} \leftrightarrow \boldsymbol{k}^{\prime}\right)
$$

where $A_{\boldsymbol{k}, \boldsymbol{p}}^{t} \equiv \int_{0}^{t} A_{\boldsymbol{k}, \boldsymbol{p}}\left(t^{\prime}\right) d t^{\prime}$. To second order,

$$
\left(\psi_{\boldsymbol{k}}^{\uparrow} \psi_{\boldsymbol{k}^{\prime}}^{\uparrow}\right)^{(2)}=\int d^{2} \boldsymbol{p} d^{2} \boldsymbol{q}\left[\left(A_{\boldsymbol{k}, \boldsymbol{p}} A_{\boldsymbol{k}^{\prime}, \boldsymbol{q}}^{t}\right)^{t}\left(\psi_{\boldsymbol{p}}^{\uparrow} \psi_{\boldsymbol{q}}^{\uparrow}\right)^{(0)}+\left(A_{\boldsymbol{k}, \boldsymbol{p}} A_{\boldsymbol{p}, \boldsymbol{q}}^{t}\right)^{t}\left(\psi_{\boldsymbol{k}^{\prime}}^{\uparrow} \psi_{\boldsymbol{q}}^{\uparrow}\right)^{(0)}\right]+\left(\boldsymbol{k} \leftrightarrow \boldsymbol{k}^{\prime}\right) .
$$

The sum of these last three equations is directly analogous to equation (A4) for the simple oscillator. Using the same reasoning here as we did for the oscillator in deriving equation (A8), and assuming that the correlation time $\left(\Lambda / v_{\mathrm{A}}\right)$ is short, we take the

\footnotetext{
${ }^{25}$ Since $\boldsymbol{w}^{\uparrow}$ is real, $\boldsymbol{w}_{\boldsymbol{k}}^{\uparrow}=\left(\boldsymbol{w}_{-\boldsymbol{k}}^{\uparrow}\right)^{*}$, where $*$ denotes the complex conjugate, and so $\psi_{\boldsymbol{k}}^{\uparrow}=\left(\psi_{-\boldsymbol{k}}^{\uparrow}\right)^{*}$; similarly, $\psi_{\boldsymbol{k}}^{\downarrow}=\left(\psi_{-\boldsymbol{k}}^{\downarrow}\right)^{*}$. It follows that $A_{\boldsymbol{k}, \boldsymbol{p}}=A_{-\boldsymbol{k},-\boldsymbol{p}}^{*}$ and $a_{\boldsymbol{k}, \boldsymbol{p}}=a_{-\boldsymbol{k},-p}^{*}$. The differential energy in up-waves within the $k$-space area $d^{2} \boldsymbol{k}$ is proportional to $d^{2} \boldsymbol{k}\left|\boldsymbol{w}_{\boldsymbol{k}}^{\dagger}\right|^{2}=d^{2} \boldsymbol{k}\left|\psi_{\boldsymbol{k}}^{\dagger}\right|^{2}$. Conservation of up-wave energy necessitates $A_{k, p}=-A_{p, k}^{*}$ and $a_{k, p}=-a_{p, k}^{*}$, as can be verified from eqs. (A14) and (A15).
} 
time derivative of the expected value of equation (A21); we get, after setting $\left\langle A A^{t}\right\rangle=\left(\Lambda / v_{\mathrm{A}}\right)\langle A A\rangle$ (with the appropriate subscripts on $A$ ), and after using equation (A16) and integrating out the delta functions on the right-hand side:

$$
\delta\left(\boldsymbol{k}+\boldsymbol{k}^{\prime}\right) \frac{d}{d t} e^{\uparrow}(\boldsymbol{k}, t)=\frac{\Lambda}{v_{\mathrm{A}}} \int d^{2} \boldsymbol{p}\left[\left\langle A_{\boldsymbol{k}, \boldsymbol{p}} A_{\boldsymbol{k}^{\prime},-\boldsymbol{p}}\right\rangle e^{\uparrow}(\boldsymbol{p}, t)+\left\langle A_{\boldsymbol{k}, \boldsymbol{p}} A_{\boldsymbol{p},-\boldsymbol{k}^{\prime}}\right\rangle e^{\uparrow}\left(\boldsymbol{k}^{\prime}, t\right)\right]+\left(\boldsymbol{k} \leftrightarrow \boldsymbol{k}^{\prime}\right) .
$$

We reexpress this equation by using equations (A14) and (A17) and the relations $a_{\boldsymbol{k}, \boldsymbol{p}}=a_{-\boldsymbol{k},-\boldsymbol{p}}^{*}, a_{\boldsymbol{k}, \boldsymbol{p}}=-a_{\boldsymbol{p}, \boldsymbol{k}}^{*}$ (from footnote $25)$, and $e^{\uparrow}(\boldsymbol{k}, t)=e^{\uparrow}(-\boldsymbol{k}, t)$ :

$$
\frac{d}{d t} e^{\uparrow}(\boldsymbol{k}, t)=2 \frac{\Lambda}{v_{\mathrm{A}}} \int d^{2} \boldsymbol{p}\left[e^{\uparrow}(\boldsymbol{p}, t)-e^{\uparrow}(\boldsymbol{k}, t)\right]\left|a_{\boldsymbol{k}, \boldsymbol{p}}\right|^{2} e^{\downarrow}(\boldsymbol{k}-\boldsymbol{p}, t) .
$$

Since each upgoing plane with fixed $z^{\uparrow}$ interacts with many statistically independent downgoing wavepackets before cascading, it is reasonable to assume isotropy in planes transverse to $\hat{z}$; after inserting equation (A15) for $a_{\boldsymbol{k}, \boldsymbol{p}}$ and changing variables,

$$
\frac{d}{d t} e^{\uparrow}(k, t)=\frac{\Lambda}{v_{\mathrm{A}}} k^{2} \int_{0}^{\infty} d k_{2} k_{2}^{3}\left[e^{\uparrow}\left(k_{2}, t\right)-e^{\uparrow}(k, t)\right] \int_{0}^{2 \pi} d \theta \sin ^{2} \theta \cos ^{2} \theta \frac{e \downarrow}{k_{1}^{2}}
$$

where

$$
k_{1} \equiv\left(k^{2}+k_{2}^{2}-2 k k_{2} \cos \theta\right)^{1 / 2} .
$$

We have redefined $\Lambda$ to absorb the factor of $4 \pi^{3}$, i.e., $\Lambda /\left(4 \pi^{3}\right) \rightarrow \Lambda$. Thus far, we have only considered a single plane with fixed $z^{\uparrow}$. If we assume that the turbulence is homogeneous in $z$, the $z$-average of the above equation is trivial, since there is no explicit dependence on $z$. We can simply reinterpret angular brackets to denote $z$-averages in addition to an ensemble average.

We emphasize that our derivation of the kinetic equation (eq. [A24]) is only valid when the correlation time, $\Lambda / v_{\mathrm{A}}$, is much smaller than the cascade time, $\left(v_{\mathrm{A}} / \Lambda\right)\left(k^{4} e^{\uparrow}\right)^{-1}$. Otherwise, equation (A22) does not follow from equation (A21).

\section{A4. STEADY-STATE FLUXES}

In steady state, we set the right-hand side of equation (A24) to zero. We substitute power-law solutions, $e^{\uparrow}(k) \propto k^{-\left(3+\alpha_{\uparrow}\right)}$, $e \downarrow(k) \propto k^{-\left(3+\alpha_{\downarrow}\right)}$, in which case the right-hand side becomes

$$
\begin{gathered}
{\left[e^{\uparrow}\left(k_{0}\right) e^{\downarrow}\left(k_{0}\right) k_{0}^{6+\alpha_{\uparrow}+\alpha_{\downarrow}} \frac{\Lambda}{v_{\mathrm{A}}}\right] k^{2} \int_{0}^{k} d k_{2} \int_{0}^{2 \pi} d \theta k_{2}^{3}\left(k_{2}^{-\left(3+\alpha_{\uparrow}\right)}-k^{-\left(3+\alpha_{\uparrow}\right)}\right)\left[1-\left(k_{2} / k\right)^{\alpha_{\uparrow}+\alpha_{\downarrow}}\right]} \\
\times \sin ^{2} \theta \cos ^{2} \theta\left(k^{2}+k_{2}^{2}-2 k k_{2} \cos \theta\right)^{-\left(5+\alpha_{\downarrow}\right) / 2} .
\end{gathered}
$$

Note that the initial square-bracketed term is independent of $k_{0}$. The above expression follows after breaking the $k_{2}$ integral into two pieces: one from 0 to $k$, and the second from $k$ to $\infty$. Then, the change of variables $k_{2} \rightarrow k^{2} / k_{2}$ is made in the second piece (a "Zakharov transformation"), so that its limits are now from 0 to $k$. Finally, this second piece is combined with the first.

In steady state, equation (A26) must vanish, so

$$
\alpha_{\downarrow}=-\alpha_{\uparrow} .
$$

Since $w_{\lambda}^{\uparrow} \sim\left(k^{2} e^{\uparrow}\right)^{1 / 2} \propto k^{-\left(1+\alpha_{\uparrow}\right) / 2}$, and similarly for $w_{\lambda}^{\downarrow}$, equation (A27) is equivalent to $w_{\lambda}^{\uparrow} w_{\lambda}^{\downarrow} \propto \lambda$ (eq. [19]). The vanishing of $\partial_{t} e \downarrow$ in steady state yields the same relation as equation (A27), and so does not give new information.

The flux associated with $e^{\uparrow}$ is given by integrating the right-hand side of equation (A24) over $d^{2} \boldsymbol{k} /(2 \pi)$ from a particular $k$ to infinity. The steady-state flux is thus given by integrating equation (A26), with $\alpha_{\downarrow}=-\alpha_{\uparrow}$. The result of the flux integration is

$$
\epsilon^{\uparrow}=f\left(\alpha_{\uparrow}\right)\left[e^{\uparrow}\left(k_{0}\right) e^{\downarrow}\left(k_{0}\right) k_{0}^{6} P O \frac{\Lambda}{v_{\mathrm{A}}}\right],
$$

where $f\left(\alpha_{\uparrow}\right)$ is a dimensionless function of $\alpha_{\uparrow}$ :

$$
f\left(\alpha_{\uparrow}\right)=\int_{0}^{1} d x x^{3} \ln x\left(x^{-\left(3+\alpha_{\uparrow}\right)}-1\right) \int_{0}^{2 \pi} d \theta \sin ^{2} \theta \cos ^{2} \theta\left(1+x^{2}-2 x \cos \theta\right)^{-\left(5-\alpha_{\uparrow}\right) / 2} .
$$

A technical note: although equation (A26) vanishes in steady state, its integral over $d^{2} \boldsymbol{k}$ gives a factor of $\alpha_{\uparrow}+\alpha_{\downarrow}$ in the denominator, which also vanishes in steady state. This $0 / 0$ ambiguity can be resolved by considering the limit as $\alpha_{\uparrow}+\alpha_{\downarrow} \rightarrow 0$ (L'Hôpital's rule).

Equation (A28) and the corresponding equation for $\epsilon \downarrow$ are equivalent to those in the heuristic discussion (eqs. [29] and [30]).

Galtier et al. (2000) plot the function $f\left(\alpha_{\uparrow}\right)$ after numerically integrating the steady-state flux integral. ${ }^{26}$ They show that $-1<\alpha_{\uparrow}<1$, that the steeper spectrum always carries more flux [i.e., $f\left(\alpha_{\uparrow}\right) / f\left(-\alpha_{\uparrow}\right)$ is a monotonically increasing function of $\left.\alpha_{\uparrow}\right]$, and that in the limit that $\alpha_{\uparrow} \rightarrow 1, \epsilon^{\uparrow} / \epsilon^{\downarrow} \rightarrow \infty$. (Clearly, this also implies that in the limit $\alpha_{\uparrow} \rightarrow-1, \epsilon^{\uparrow} / \epsilon^{\downarrow} \rightarrow 0$.) In $\S 4.3 .1$,

\footnotetext{
${ }^{26}$ More precisely, their Figure 2 is proportional to $\left[f\left(\alpha_{\uparrow}\right) f\left(-\alpha_{\uparrow}\right)\right]^{-1 / 4}$, and their Figure 3 shows $f\left(\alpha_{\uparrow}\right) / f\left(-\alpha_{\uparrow}\right)$.
} 
we explain the physical reason why $-1<\alpha_{\uparrow}<1$. When this condition is violated, the cascade becomes nonlocal. Galtier et al. (2000) find infinite fluxes when these inequalities are saturated because they consider an infinitely extended spectrum, which leads to unphysical results when the cascade is nonlocal.

As discussed in $\S 4.2$, in steady state we are primarily concerned with the case in which the up- and downgoing fluxes are comparable, so $\left|\alpha_{\uparrow}\right| \ll 1$. In this limit, we linearize $f$ about $\alpha_{\uparrow}=0$, yielding approximately

$$
f\left(\alpha_{\uparrow}\right) \simeq f(0)\left(1+0.5 \alpha_{\uparrow}\right), \quad\left|\alpha_{\uparrow}\right| \ll 1,
$$

after numerical integrations of equation (A29). This result is used in $\S 4.2$ to calculate the energy spectra given the fluxes $\epsilon^{\uparrow}$ and $\epsilon^{\downarrow}$. Although the value $f(0)$ is much less important than the dependence of $f$ on $\alpha_{\uparrow}$, we use it when discussing the results of our numerical simulations; it is given by

$$
f(0) \simeq 1.87
$$

\section{A5. THE ZERO-FREQUENCY MODE}

Weak MHD turbulence is often described as being based on three-wave resonances, in which one of the three waves has zero frequency (e.g., Shebalin et al. 1983; Galtier et al. 2000, 2002). However, the interpretation of a zero-frequency wave is unclear. After all, it should require an infinite amount of time for three interacting waves to "realize" that one of them has zero frequency. In this subsection, we clarify the role of the zero-frequency mode. In the process, we compare our derivation of the kinetic equation with that of Galtier et al. (2002). Note that in our derivation in $\S$ A.3 we avoided discussing Fourier wavemodes, since we did not Fourier transform in $t$ or in $z$.

The evolution of the up-waves at fixed $z^{\uparrow} \equiv z-v_{\mathrm{A}} t$ is given in equation (A18). However, for clarity, in this subsection we consider the random oscillator instead (eq. [A2]):

$$
d \psi / d t=i A \psi .
$$

It is a simple matter to extend our discussion to weak MHD turbulence by replacing $\psi$ with $\psi_{\boldsymbol{k}}^{\uparrow} \psi_{\boldsymbol{k}^{\prime}}^{\uparrow}$, replacing $i A$ with $A_{\boldsymbol{k}, p}$, and summing over the appropriate indices.

In $\S$ A. 2 we derived the equation for $\langle\psi\rangle$, assuming that the cascade time of $\langle\psi\rangle$ is longer than the correlation time of $A$ :

$$
d\langle\psi\rangle / d t=-\left\langle A A^{t}\right\rangle\langle\psi\rangle,
$$

where $\left\langle A A^{t}\right\rangle \equiv \int_{t-T}^{t}\left\langle A(t) A\left(t^{\prime}\right)\right\rangle d t^{\prime} \sim A_{\text {rms }}^{2} \tau_{\text {corr }}$; the value of $T$ is unimportant as long as $T>\tau_{\text {corr }}$.

We can see how the zero-frequency mode enters by rewriting $\left\langle A A^{t}\right\rangle$ in terms of $\hat{A}_{\omega}$, the Fourier transform of $A$ :

$$
\left\langle A A^{t}\right\rangle=\int_{-\infty}^{\infty} \frac{d \omega^{\prime}}{2 \pi} \int_{-\infty}^{\infty} \frac{d \omega}{2 \pi}\left\langle\hat{A}_{\omega^{\prime}} \hat{A}_{\omega}\right\rangle e^{i \omega^{\prime} t} \int_{t-T}^{t} e^{i \omega t^{\prime}} d t^{\prime}
$$

To compare this result with that of Galtier et al. (2002), we use similar notation. We denote the power spectrum of $\hat{A}_{\omega}$ by $q_{\omega}$ :

$$
\left\langle\hat{A}_{\omega^{\prime}} \hat{A}_{\omega}\right\rangle \equiv q_{\omega} \delta\left(\omega^{\prime}+\omega\right) ;
$$

the Dirac delta function results from the time invariance of the statistical properties of $A$. We also define

$$
\Delta(\omega) \equiv \frac{1}{i \omega} e^{i \omega t}\left(1-e^{i \omega T}\right) .
$$

With these two definitions, equation (A34) is

$$
\left\langle A A^{t}\right\rangle=\int_{-\infty}^{\infty} \frac{d \omega}{4 \pi^{2}} q_{\omega}\left[e^{-i \omega t} \Delta(\omega)\right] .
$$

If this equation is inserted into equation (A33), the resulting equation is equivalent to equation (7) in Galtier et al. (2002). As these authors argue, when $T \rightarrow \infty, \exp (-i \omega t) \Delta(\omega) \rightarrow \pi \delta(\omega)$, so

$$
\left\langle A A^{t}\right\rangle=\int_{-\infty}^{\infty} \frac{d \omega}{4 \pi} q_{\omega} \delta(\omega)
$$

which corresponds to equation (8) in Galtier et al. (2002). From this expression, we can see why the zero-frequency mode enters: it is simply a consequence of the term $\left\langle A A^{t}\right\rangle$. It does not require an infinite time for $\langle\psi\rangle$ to interact with $q_{\omega=0}$; rather, the only quantity that enters into $q_{\omega=0}$ is the value of $A$ within the time $\tau_{\text {corr }}$ of $t$. We can see this more clearly by explicitly writing the expression for $q_{\omega}$. Of course, the power spectrum $q_{\omega}$ is simply the Fourier transform of the correlation function of $A$ :

$$
q_{\omega}=4 \pi \int_{-\infty}^{0} e^{-i \omega \tau}\langle A(t) A(t+\tau)\rangle d \tau
$$

As long as $\omega \lesssim 1 / \tau_{\text {corr }}$, we have $q_{\omega} \simeq 4 \pi\left\langle A A^{t}\right\rangle$. 
One of our reasons for discussing the Fourier-space picture in detail is that there have been a large number of confusing remarks about it in the literature. For example, Galtier et al. $(2000,2002)$ claim that, since the kinetic equation is apparently not applicable to the zero-frequency mode, there might be a "condensation" of zero-frequency modes. Since the zerofrequency mode is so important, this condensation might have dramatic implications for the cascade. However, as long as correlation times are shorter than cascade times, the kinetic equation gives a complete description of the turbulence, and is applicable to the zero-frequency mode as well, which is simply given by $q_{\omega=0}=4 \pi\left\langle A A^{t}\right\rangle$. For this not to be true, large-time correlations would have to build up in $A$ (see eq. [A39]). Since $A$ corresponds to $\psi \downarrow$ in weak MHD turbulence, large-time correlations at fixed $z^{\uparrow}$ correspond to large-distance correlations along the $z$-direction. But it is impossible for two downgoing wavepackets, initially uncorrelated, to become correlated. This is because the equation for their evolution is linear: recall that in weak turbulence the back-reaction term is negligible.

Biskamp, D. 1995, Nonlinear Magnetohydrodynamics (Cambridge: Cambridge Univ. Press)

. 2000, Magnetic Reconnection in Plasmas (Cambridge: Cambridge Univ. Press)

Biskamp, D., \& Müller, W.-C. 2000, Phys. Plasmas, 7, 4889

Borue, V., \& Orszag, S. A. 1995, Europhys. Lett., 29, 687

Cho, J., Lazarian, A., \& Vishniac, E. 2002, ApJ, 564, 291

Cho, J., \& Vishniac, E. T. 2000, ApJ, 539, 273

Dobrowolny, M., Mangeney, A., \& Veltri, P. 1980, Phys. Rev. Lett., 45, 144

Falkovich, G. 1994, Phys. Fluids, 6, 1411

Galtier, S., Nazarenko, S. V., Newell, A. C., \& Pouquet, A. 2000, J. Plasma Phys., 63, 447 2002, ApJ, 564, L49

Goldreich, P., \& Sridhar, S. 1995, ApJ, 438, 763 1997, ApJ, 485, 680

Grappin, R., Pouquet, A., \& Léorat, J. 1983, A\&A, 126, 51

\section{EFERENCES}

Iroshnikov, P. 1963, Soviet Astron., 7, 566

Kraichnan, R. 1965, Phys. Fluids, 8, 1385

Lithwick, Y., \& Goldreich, P. 2001, ApJ, 562, 279

Maron, J., \& Goldreich, P. 2001, ApJ, 554, 1175

Montgomery, D., \& Matthaeus, W. H. 1995, ApJ, 447, 706

Montgomery, D., \& Turner, L. 1981, Phys. Fluids, 24, 825

Müller, W., \& Biskamp, D. 2000, Phys. Rev. Lett., 84, 475

Ng, C. S., \& Bhattacharjee, A. 1996, ApJ, 465, 845 1997, Phys. Plasmas, 4, 605

Shebalin, J. V., Matthaeus, W. H., \& Montgomery, D. 1983, J. Plasma Phys., 29, 525

Sridhar, S., \& Goldreich, P. 1994, ApJ, 432, 612

van Kampen, N. G. 1992, Stochastic Processes in Physics and Chemistry (Amsterdam: Elsevier), Chap. XVI

Zakharov, V. E., L'vov, V. S., \& Falkovich, G. 1992, Kolmogorov Spectra of Turbulence I (Berlin: Springer) 